\title{
含氟药物研究进展和芳(杂)环氟化及 $\mathrm{N}(n=1,2,3)$ 氟甲基化新方法
}

\author{
张 雯* 金传飞 张英俊 \\ (广东东阳光集团 药业研究院 东莞 523871)
}

\begin{abstract}
摘要 论述了最近几年含氟药物的最新研究成果和含氟新药的研究概况, 从药物化学工作者的角度对芳(杂环)的氟化 反应，氟甲基化、二氟甲基化和三氟甲基化反应进展和亮点做了分析归纳和总结，对一些金属催化的氟化新反应的机 理进行了简单的阐述. 指出药物结构优化中充分利用氟化学的最新研究成果是一条值得探索的捷径.
\end{abstract}

关键词 氟；含氟药物；氟化反应；三氟甲基化；二氟甲基化；一氟甲基化；药物设计

\section{Recent Advances in Research and Development of Fluorinated Drugs and New Methods for Fluorination, Mono-, Di- and Tri-fluoromethylation}

\author{
Zhang, Ji* Jin, Chuanfei Zhang, Yingjun \\ (HEC Pharm Group, HEC Research and Development Center, Dongguan 523871)
}

\begin{abstract}
This review outlines the recent advances of fluorinated drugs and latest research overview from the perspective of a medicinal chemist wherein the synthesis and use of trifluoromethylation, difluoromethylation, monofluoromethylation and fluorinated aromatic (heterocyclic) compounds are highlighted. It is pointed out that the full use of the latest progress in fluorine chemistry provides a shortcut to new fluorinated compounds or previously difficult to prepare compounds which is worth exploring for drug discovery.

Keywords fluorine; fluorinated drugs; fluorination; trifluoromethylation; difluoromethylation; monofluoromethylation; drug design
\end{abstract}

自从 1956 年第一次出现含三氟甲基的精神类用药 氟非那嗪(Fluphenazine)和 1957 年首次引入第一个含氟 抗癌药物 5-氟尿嘧啶(5-Fluorouracil) 以来 ${ }^{[1]}$, 半个多世 纪过去了. 近十年来, 隨着氟化学研究的进展和对氟原 子及含氟取代基的深入了解, 药物科学家正在进一步开 掘含氟药物这座新药研发中的金山银矿 (Scheme 1) ${ }^{[2]}$. 最新统计表明, 目前全球含氟药物年销售额在 400 亿美 元左右, 全球销售前 200 名的药物中, 含氟药物就占了 29 个, 销售额总计 320 亿美元. 由此看来含氟药物的应 用及研发前景相当可观, 约有 $25 \%$ 30\%的新药研发是 建立在氟化学原料产品基础之上的. 依文献报道, 约 15\% 20\%的新药都含有氟原子或三氟甲基等基团(图 $1)^{[3]}$. 据我们统计, 截止 2013 年底, 一共有 163 个含氟 药物接受被美国食品和药物管理局(FDA)的批准上市 ${ }^{[4]}$, 这充分说明氟原子是除氯原子之外, 第二个最令药物化
学工作者喜欢的卤素原子. 在药物研究中, 充分利用构 效关系(SAR)的方法学探索和越来越多含氟中间体的可 得性, 都为含氟药物的研究提供了巨大的推动. 友容置 疑, 最近几年的有机氟化学的研究热潮必将为新一波的 新药探寻提供更多的方法和手段, 而在氟化学领域有世 界一流的中国研究团队的参与 ${ }^{[5]}$, 也必将会浓墨重彩地 书写药物研发的崭新一页. 本文将近年来这一领域的研 究做一简述概括, 希望能为现代药物的合成研发提供帮 助 ${ }^{[6]}$.

不同于其它的卤素，氟原子由于其独特的电子结 构, 它具有最强的电负性和与氢原子一般大小的原子半 径, 因而也能更加方便合理地取代氢原子而进行药物分 子结构的微调和修饰，阻断易代谢位点从而改变药物代 谢的途径及代谢速度; 并通过分子间氢键的作用, 延长 药物在体内的作用时间, 提高药物的生物利用度和选择

*E-mail: zhangji@hecpharm.com

Received October 29, 2013; revised December 9, 2013; published online December 19, 2013.

Project supported by the Introduction of Innovative R\&D Team Program of Guangdong Province.

广东省引进创新科研团队计划资助项目. 
<smiles>O=c1[nH]cc(F)c(=O)[nH]1</smiles>

5-Fluorouracil 抗癌 Fluphenazine 精神类用药<smiles>CC(C)c1c(C(=O)Nc2ccccc2)c(-c2ccccc2)c(-c2ccc(F)cc2)n1CC[C@H](O)C[C@H](O)CC(=O)O</smiles>

Atorvastatin 降胆固醇

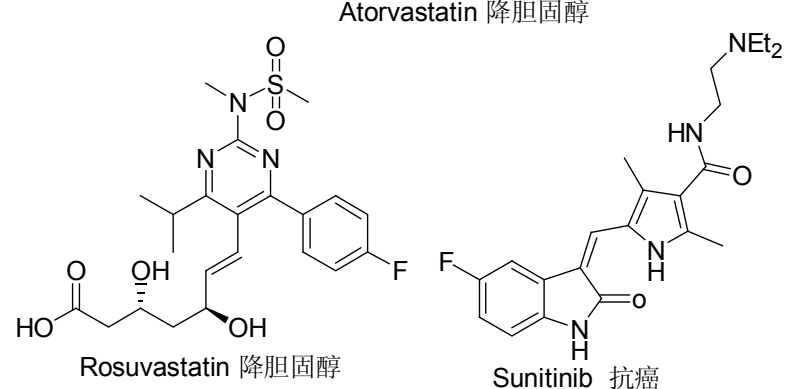<smiles>N[C@@H](CC(=O)N1CCn2c(nnc2C(F)(F)F)C1)Cc1cc(F)c(F)cc1F</smiles>

Scheme 1

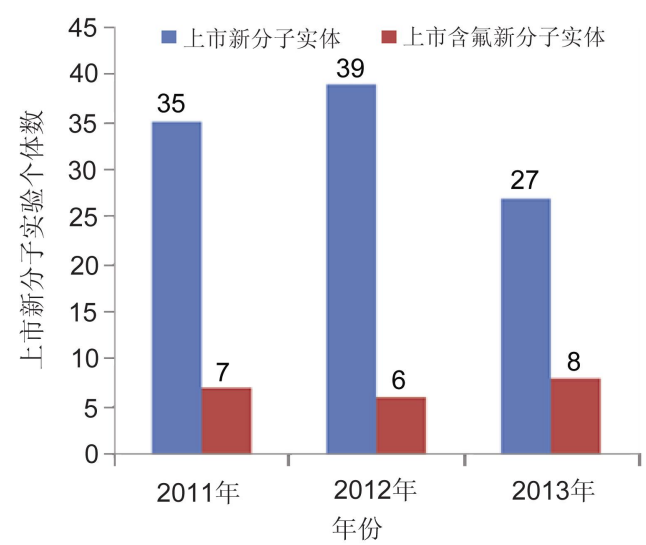

图 1 2011 2013 美国食品和药物管理局批准上市新药

Figure 1 2011 2013 FDA approval drugs

性. 最值得一提的是, 三氟甲基由于具有很强的吸电子 性、亲脂性和稳定性等特点, 具有很强的疏水性而表现 出理想的脂溶性, 具有更好的生物通透性和靶向选择 性, 因此在许多上市药物和临床药物常常含有三氟甲基 的芳环或杂环分子 ${ }^{[7]}$.

2011 年美国 FDA 共批准了 35 个小分子化学药物, 其中有 7 个是含氟新分子实体(Scheme 2)；2012 年共批
准了 33 个小分子化学药物, 其中有 6 个为含氟有机分子 (Scheme 3)；2013 年一共有 8 个含氟药物获批(Scheme 4). 据不完全统计, 总共有数十个含氟药物进入了临床 研究, 其中一些代表性含氟药物见 Scheme 5, 如 Merck 临床三期的胆固醇转运蛋白(CEPT)抑制剂 Anacetrapib 和 Lilly 的 Evacetrapib, 这两个临床药物都含有三氟甲 基和氟的芳香烃结构单元, 预期是药物中的重磅炸弹 (Blockbuster drugs). 另外 Daiichi 研发的高效鲨烯合成 酶抑制剂 DF-461 据称效果比上市的 HMG- CoA 还原酶 抑制剂 Atorvastatin(Lipitor)还要好. 可见含氟药物的研 究正处于一个相当令人鼓舞的发展局面 ${ }^{[8]}$, 而有机氟化 学的突破为这一药物研发的加速提供了很好的帮助.<smiles>CCCS(=O)(=O)Nc1ccc(F)c(C(=O)c2c[nH]c3ncc(-c4ccc(Cl)cc4)cc23)c1F</smiles>

Vemurafenib 黑色素瘤<smiles>C[C@@H](Oc1c(F)ccc(Cl)c1N(C)CCN(C)C)c1cc(-c2cnn(C3CCNCC3)c2)cnc1N</smiles>

Crizotinib 非小细胞肺癌<smiles>COc1cc2c(Nc3ccc(Br)cc3F)ncnc2cc1OCC1CCN(C)CC1</smiles>

Vandetanib 非小细胞肺癌<smiles>CCOC(=O)Nc1ccc(NCc2ccc(F)cc2)cc1N</smiles>

Roflumilast 慢性阻塞性肺病

Retigabine 抗癫㾁<smiles>CCSc1nc(NC2C[C@@H]2c2ccc(F)c(F)c2)c2nnn([C@@H]3C[C@H](OCCO)[C@@H](O)[C@H]3O)c2n1</smiles>

Ticagrelor 抗血小板<smiles>CNC(=O)c1cc(Oc2ccc(NC(=O)Nc3ccc(Cl)c(C(F)(F)F)c3)cc2)ccn1</smiles>

Scheme 2 


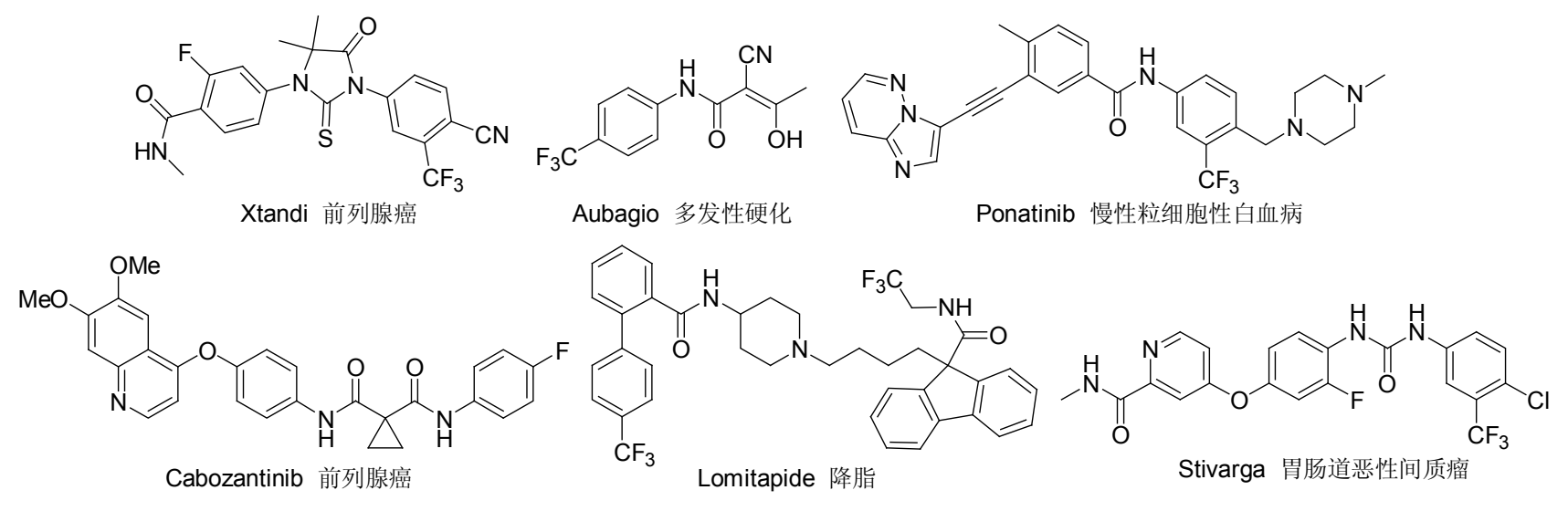

Scheme 3<smiles>Cc1ccc(Cc2csc(-c3ccc(F)cc3)c2)cc1C1OC(CO)[C@@H](O)[C@H](O)[C@H]1O</smiles>
Invokana 糖尿病<smiles>COC(=O)N(C)c1c(N)nc(-c2nn(Cc3ccccc3F)c3ncccc23)nc1N</smiles>

Riociguat 治疗肺动脉高压<smiles>CC(C)(C)c1nc(-c2cccc(NS(=O)(=O)c3c(F)cccc3F)c2F)c(-c2ccnc(N)n2)s1</smiles>

Tafinlar 黑色素瘤<smiles>CC(=O)Nc1cccc(-n2c(=O)n(C3CC3)c(=O)c3c(Nc4ccc(I)cc4F)n(C)c(=O)cc32)c1</smiles>

Mekinist 黑色素瘤<smiles>CC(C)OC(=O)C(C)NP(=O)(OCC1OC(n2ccc(=O)[nH]c2=O)C(C)C1O)Oc1ccccc1</smiles><smiles>O=[N+]([O-])c1ccc(-c2nc3ccc(O)cc3s2)cc1Br</smiles>

Flutemetamol F18 脑影像药物

Scheme 4<smiles>C#C[C@@H](CC)c1cc(CN(c2nnn(C)n2)C2CCCN(C[C@H]3CC[C@H](C(=O)O)CC3)c3c(C)cc(C)cc32)cc(C(F)(F)F)c1</smiles><smiles>COc1cc(F)c(C(C)C)cc1-c1ccc(C(F)(F)F)cc1CN1C(=O)OC(c2cc(C(F)(F)F)cc(C(F)(F)F)c2)C1C</smiles><smiles>COc1cccc(C2OC(CC(=O)O)c3nnc(C(F)(F)F)n3-c3ccc(Cl)cc32)c1Cl</smiles>

DF-461 一期

\section{Scheme 5}

从近三年获批的含氟药物结构来看, 一共有 17 个 是氟代芳香烃, 有 6 个是含有三氟甲基的芳香环. 其中 有 2 个药物既有三氟甲基又有氟原子. 显然, 有机芳香 烃的氟化反应对新药研发是极其重要的, 为此, 本文就 近几年来芳(杂)环氟化及 $\mathrm{N}(n=1,2,3)$ 氟甲基化的研究 进展和亮点并结合实例进行分析总结.

\section{1 芳烃和杂芳烃氟化反应(2006～2013)}

芳烃和杂芳烃的氟化反应可分为亲电取代(正离子 $\mathrm{F}^{+}$试剂)和亲核取代(负离子 $\mathrm{F}^{-}$试剂)两类型. 氟化反应 少不了使用氟化试剂，而且一般都使用过量的. 许多氟 化试剂，包括能提供氟正离子和氟负离子的氟化物见 Scheme 6, 除了常用的 KF, 一般氟化试剂都不便宜, 尤 


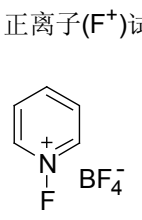

简称: $[\mathrm{pyF}] \mathrm{BF}_{4}$<smiles>F[n+]1c(Cl)cccc1Cl</smiles>

简称: $\left[\mathrm{Cl}_{2}\right.$ pyF $] \mathrm{OTf}$

负离子 $\left(F^{-}\right)$试剂

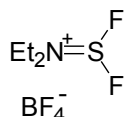

简称：Xtalfluor-E

$\mathrm{AgF} \quad \mathrm{CsF}$

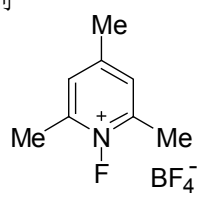

$\left[\mathrm{Me}_{3} \mathrm{pyF}\right] \mathrm{BF}_{4}$<smiles>F[n+]1ccccc1</smiles>

[pyF]OTf<smiles>Cc1cc(C)[n+](F)c([N+](=O)[O-])c1</smiles>

$\left[\mathrm{Me}_{3}\right.$ pyF]OTf (NFTPT)<smiles>FC1(Br)CN2CC[N+]1(CCl)CC2</smiles>
F-TEDA-BF 4 F-TEDA-BF
(Selectfluor)<smiles>Cc1cc(C)[n+](F)c(C)c1</smiles><smiles>O=S(=O)(c1ccccc1)N(F)S(=O)(=O)c1ccccc1</smiles>

$\left[\mathrm{Me}_{3} \mathrm{pyF}_{\mathrm{PF}} \mathrm{F}_{6}\right.$

NFSI<smiles>FC(F)(F)F</smiles>

F-TEDA-PF 6<smiles>O[N+]12CCN(CC1)C(CBr)C2</smiles>

$\mathrm{F}$

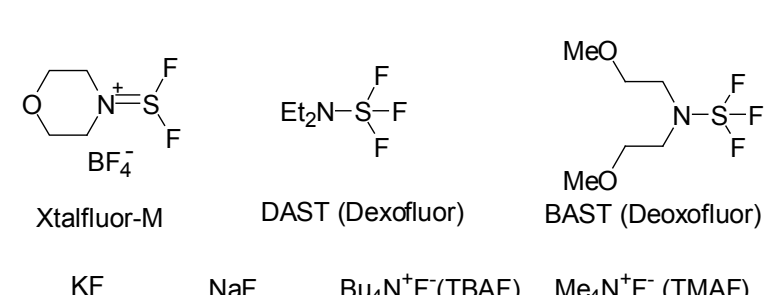

Scheme 6

其是一些制备困难的有机氟试剂，价格尤为昂贵.

\section{1 利用含氮(胺基、酰胺等)芳(杂)环为原料}

使用含氮杂环的联苯衍生物, Sanford 小组 ${ }^{[9]}$ 在 2006 年首先发展了有效的氟化反应(Eq. 1), 在 $10 \mathrm{~mol} \%$ $\mathrm{Pd}(\mathrm{OAc})_{2}$ 存在的条件下, 选择性地引入了氟原子. 这也 是在钯(II)催化下, 温和氧化条件下利用亲电的氟化试 剂 $\left(\mathrm{F}^{+}\right.$, 不同于使用亲核氟试剂 $\left.\mathrm{F}^{-}\right)$成功进行的经由直接 $\mathrm{C}-\mathrm{H}$ 活化、氧化氟化(Oxidative fluorination)的创新反应.

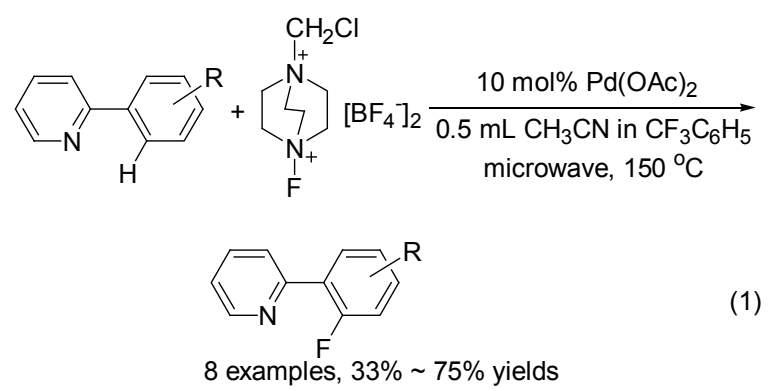

2009 年, Scripps 的 $\mathrm{Yu}$ 等 ${ }^{[10]}$ 利用钯盐催化的、邻位 导向基诱导的 $\mathrm{C}-\mathrm{H}$ 活化策略, 用 $\mathrm{F}^{+}$试剂在 NMP 为促 进剂的情况下有效地制备了邻位含氟的芳香化合物(Eq. 2).

利用导向基的弱配位作用, $\mathrm{Yu}$ 小组 ${ }^{[11]}$ 成功利用了 $\mathrm{Pd}$ 催化下的 $\mathrm{C}-\mathrm{H}$ 活化导向基的邻位, 有效地进行苯甲 酸衍生物的单氟化和双氟化(Scheme 7), 显然这是一个 有 $\mathrm{F}^{+}$试剂参与的反应.

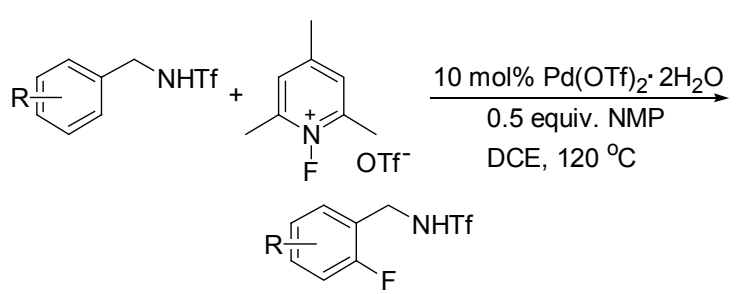

17 examples, $41 \% \sim 88 \%$ yields

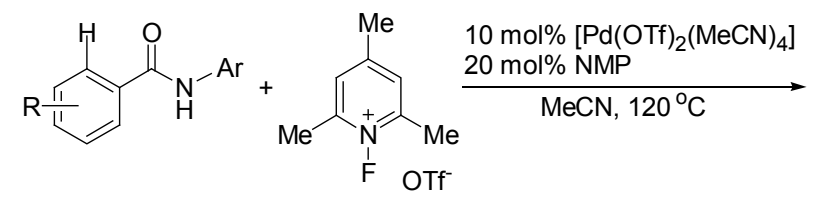<smiles>CNC(=O)c1ccccc1F</smiles>

17 examples, $36 \% \sim 78 \%$ yields

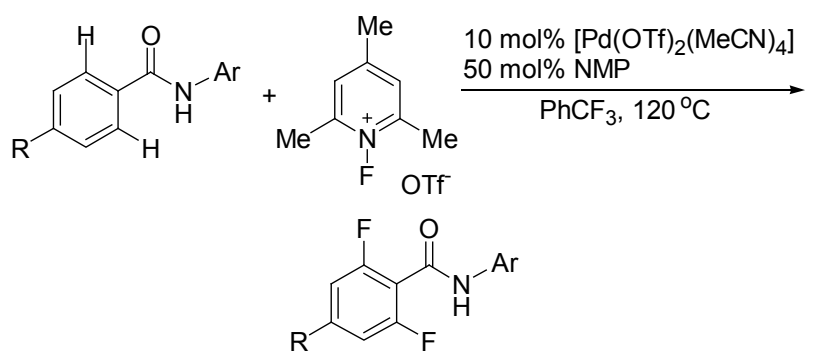

3 examples, $66 \% \sim 88 \%$ yields

\section{Scheme 7}

不难看出, 上述三个反应都是在邻位导向基(directing groups)存在下钯(II)催化的亲电氟化反应, 反应经 
历了环钯化(Cyclopalladation)和亲电氟化两过程. 巧妙 的是, 分子内氮原子从不同的形式(如吡啶中的 $\mathrm{N}$; 取代 的胺和酰胺) 作为供电子体, 参与了分子内环钯化过程, 其特点是形成有利的五元钯(II)络合物中间态(Scheme 8).

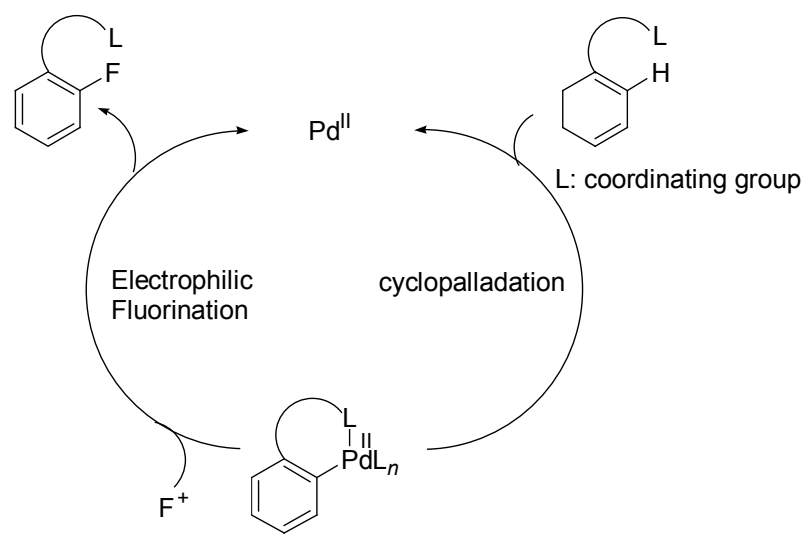

Scheme 8

在没有导向基存在的条件下，钯催化的亲电氟化反 应的机理如图所示 (Scheme 9). 首先是通过转移金属化 而形成的碳钯键, 由于不涉及到环钯化 (cyclopalladation), 也不需要导向基, 因此反应底物的多样性就更加 广泛, 缺点是底物要进行预官能团化, 必须引入适当的 基团进行转移金属化(transmetalation). 随后通过亲电氟 化试剂氧化将 $\mathrm{Pd}(\mathrm{II})$ 络合物转化为 $\operatorname{Pd}(\mathrm{IV})$ 的络合物, 进 而通过还原消除生成 $\mathrm{C}-\mathrm{F}$ 键.

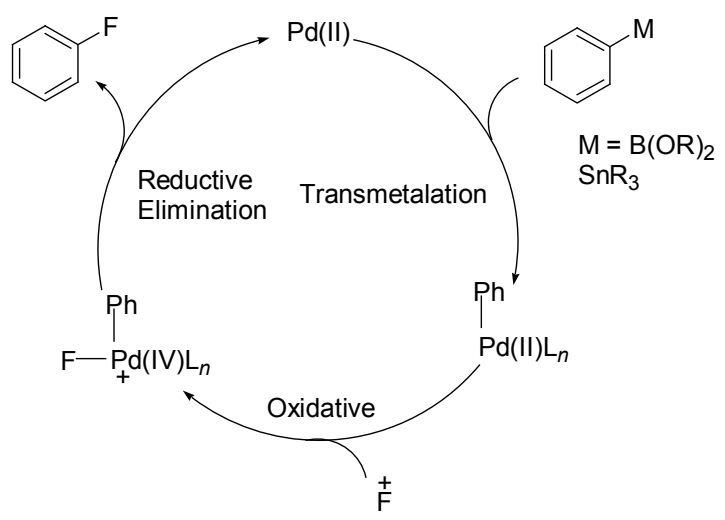

Scheme 9

通过利用导向基, Daugulis 小组 ${ }^{[12]}$ 发展了以 8-胺基 喹啉为底物的铜盐催化的, $\mathrm{AgF}$ 为氟试剂的杂芳环的邻 位氟化亲核取代反应(Scheme 10), 取得了较好的结果, 值得一提的是通过改变实验条件, 单氟化和双氟化均可 实现，反应同时显示具有优秀的官能团兼容性.

\section{2 利用芳基锡烷/有机嗍化物为原料}

利用铜盐催化, Sanford 小组 ${ }^{[13]}$ 发展了温和条件下的 芳基锡烷和三氟硼酸钾的氟化反应(Scheme 11). 反应具

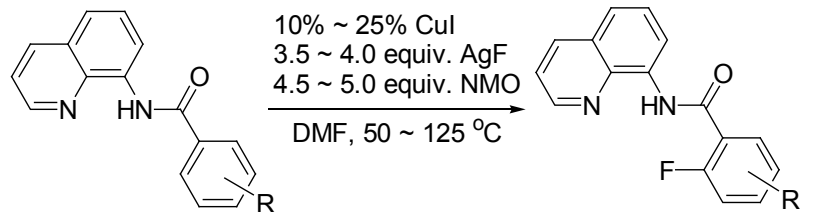

8 examples, $56 \% \sim 80 \%$ yields

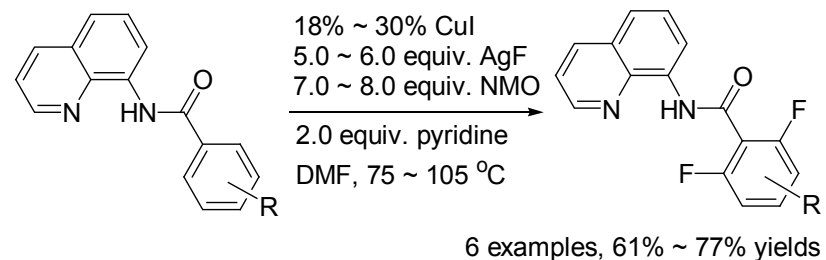

Scheme 10

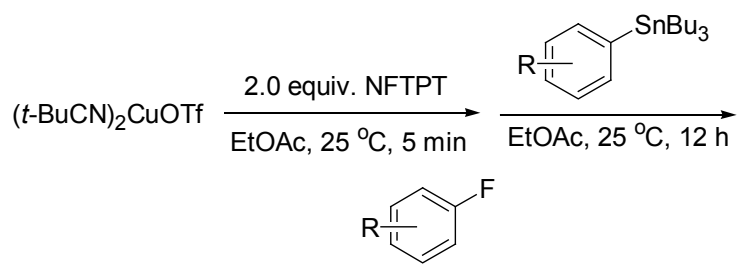

12 examples, $60 \% \sim 81 \%$ yields

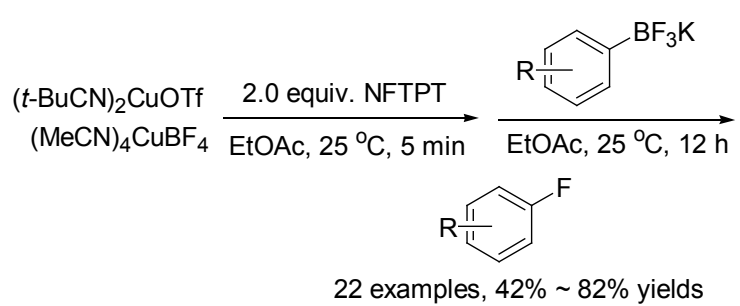

Scheme 11

有很好的官能团兼容性, 收率优良. 并且不需要使用昂 贵的金属. 反应据认为是通过 $\mathrm{Cu}(\mathrm{III})$ 中间体完成.

最近, Hartwig 小组报道 ${ }^{[14]}$ 了铜盐催化下, 利用 $\mathrm{F}^{+}$ 试剂对有机硼酸酯的氟化反应(Scheme 12). 反应条件相 对温和, 虽然铜试剂和银试剂的用量较大. 与此同时, 通过对 ${ }^{19} \mathrm{~F}$ NMR 的考查, 确证了 $\mathrm{Cu}(\mathrm{III})$ 氟络合物的存 在. 鉴于有机硼酸酯易得，这一新方法为制备氟代芳烃 又提供了一个新手段.

利用芳基有机硼酸、硼酸酯和三氟硼酸钾盐为原料, 哈佛大学的 Ritter 小组 ${ }^{[15]}$ 发展了温和条件下的钯盐(II) 催化的氟化方法并取得了优异的结果(Eq. 3). 该法操作 简单易行. 机理研究表明这是一个通过单电子转移途径 的过程，并且分离并确证钯(III)中间体的存在.

最近, Sanford 等 ${ }^{[16]}$ 报告了温和条件下 $\left(60{ }^{\circ} \mathrm{C}, 20 \mathrm{~h}\right)$, 利用 $\mathrm{Cu}(\mathrm{OTf})_{2}$ 媒促芳香硼酸钾转变为相应氟代芳香烃 的新反应 (Eq. 4). 不同于原先报道的 $\mathrm{Cu}(\mathrm{I})$ 催化、 $\mathrm{F}^{+}$进 攻的亲电反应, 这是一个 $\mathrm{Cu}(\mathrm{II})$ 参与的、 $\mathrm{F}^{-}$进攻的亲核 反应. 认为二价铜有双重作用: 一是媒促 $\mathrm{Ar}-\mathrm{F}$ 的偶联; 二是作为氧化剂参与中间体 $\mathrm{Cu}(\mathrm{III})(\mathrm{F})$ 的形成. 


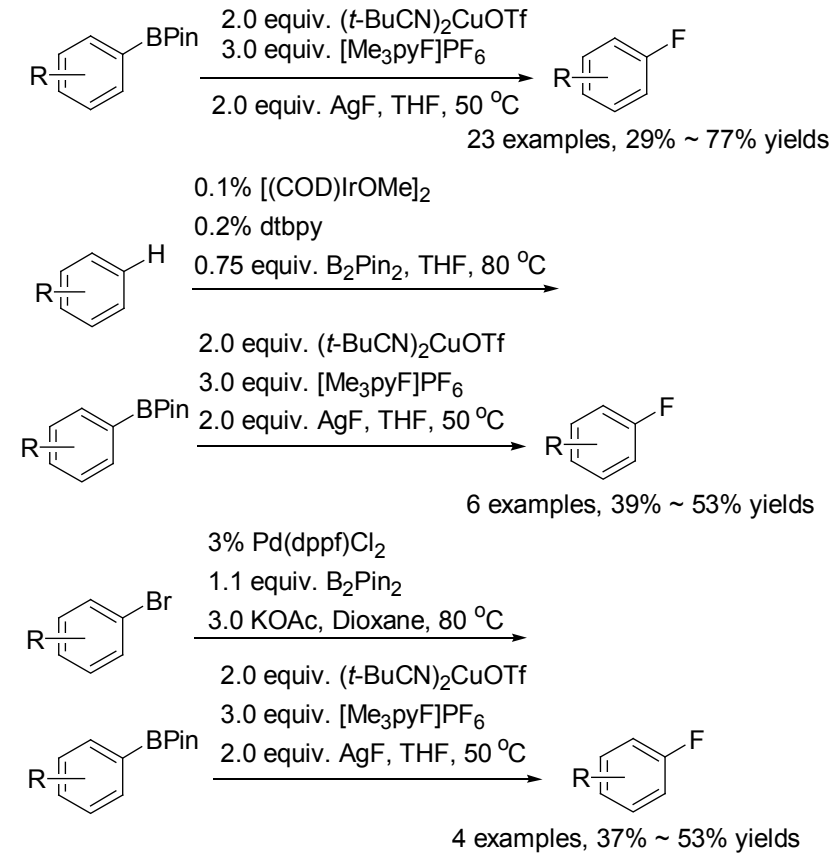

Scheme 12

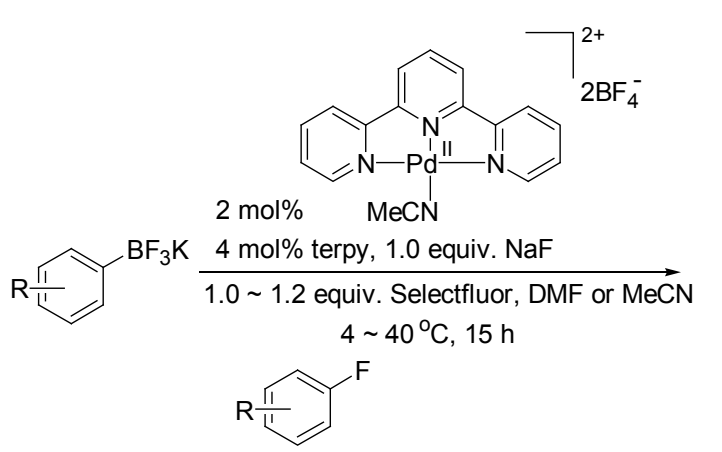

15 examples, $63 \% \sim 99 \%$ yields

$$
\text { } \underset{\mathrm{CH}_{3} \mathrm{CN}, 60^{\circ} \mathrm{C}, 20 \mathrm{~h}}{\mathrm{Ba}}
$$

17 examples, $21 \sim 71 \%$ yields

对位或邻位有富电子基团存在的有机硣酸在活泼 的亲电氟化试剂作用下, 能够较为有效地制备相应的氟 代苯，而间位有供由子基的嗍酸则完全没有反应 ${ }^{[17]}$. 2009 年 Ritter 等 ${ }^{[18]}$ 报道了银盐媒促的氟化反应，提供了 一条从有机硼酸制备氟代苯衍生物的新方法(Scheme 13).

\section{3 利用芳基锡烷为原料}

使用不同的银盐( $\left.\mathrm{AgOTf}, \mathrm{Ag}_{2} \mathrm{O}\right)$ 催化剂, Ritter 等 ${ }^{[19,20]}$ 在 2009 年和 2010 年先后首先发展了温和条件下 的芳香锡烷的氟化反应(Scheme 14), 并取得了良好的实 验结果. 这一新合成方法对有效制备 ${ }^{18} \mathrm{~F}$ 同位素标记化 合物用于 PET(正电子发射断层扫描技术)成像技术是非 常重要的. PET 现已成为诊断和指导治疗肿瘤、冠心病

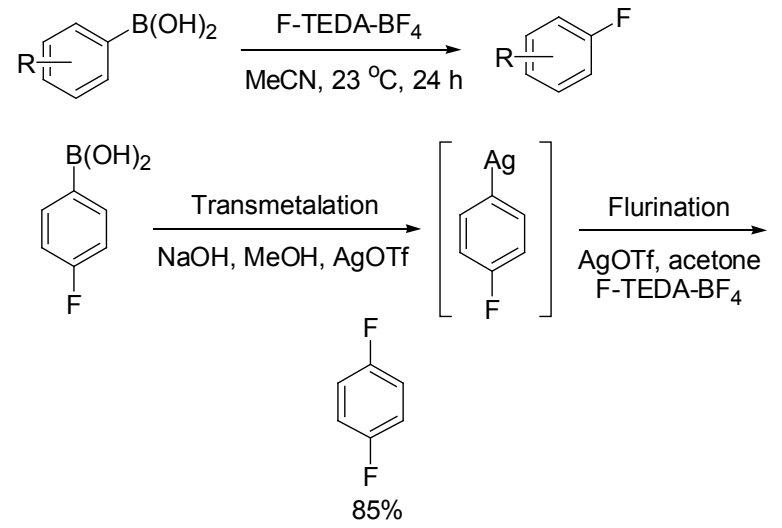

Scheme 13

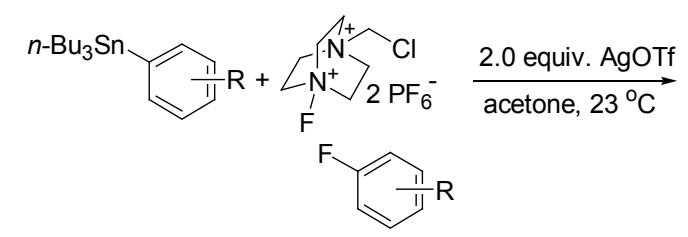

12 examples, $63 \% \sim 83 \%$ yields

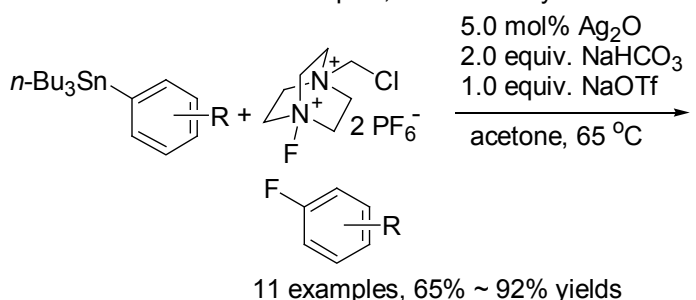

Scheme 14

和心脑血管疾病等威胁人类生命的最佳手段, 虽然有许 多手段可发射正电子的同位素，但在临床医学上 ${ }^{18} \mathrm{~F}$ 是 最合适的放射性同位素. 这也是近几年来，将氟原子引 入芳环研究红红火火的主要原因之一.

\section{4 利用芳香碘化物为原料和卤素交换反应 (Halex processes)}

Hartwig 小组 ${ }^{[21]}$ 发现在铜盐和 $\mathrm{AgF}$ 的作用下, 取代 碘苯能够有效地进行卤素交换而制备相应的氟苯 (Scheme 15). 据机理研究表明, 该反应有 $\mathrm{Cu}(0)$ 和 $\mathrm{CuF}_{2}$ 的形成. 反应没有完成催化循环.

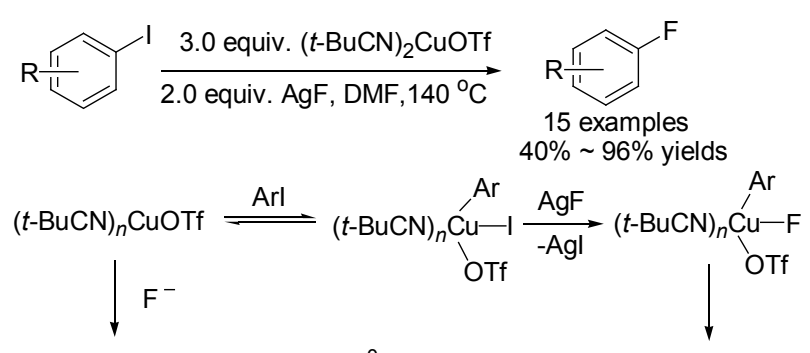

$(t-\mathrm{BuCN})_{n} \mathrm{CuOF} \longrightarrow 1 / 2\left(\mathrm{Cu}^{0}+\mathrm{CuF}_{2}\right)$

$\mathrm{ArF}+(t-\mathrm{BuCN})_{n} \mathrm{CuOTf}$

\section{Scheme 15}

使用 Halex 即卤素交换反应也是制备氟代芳烃的一 
种方法. 例如在 DMSO 为溶剂下使用无水的四甲基氟 化铵(TMAF), 在非常温和的条件下 $\left(20{ }^{\circ} \mathrm{C}, 1.5 \mathrm{~h}\right)$ 能够非 常迅速并高效地从 2,6-二氯吡啶中制备 2,6-二氟吡啶 (Scheme 16 $)^{[22]}$. 而先前的合成方法条件相当苛刻, 且产 率一般 $(56 \%)^{[23]}$.

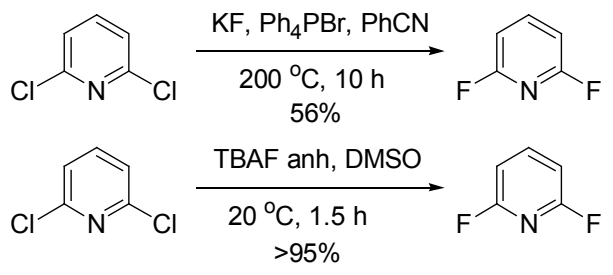

Scheme 16

值得一提的是在无水 DMSO 为溶剂的条件下, 使 用 TMAF 以溴苯和 2-溴荎为原料, 能够方便地制备氟苯 和 2-二氟荎及 3-氟荎(Scheme 17) $)^{[24]}$. 显然这是通过芳炔 活泼中间体实现的过程.

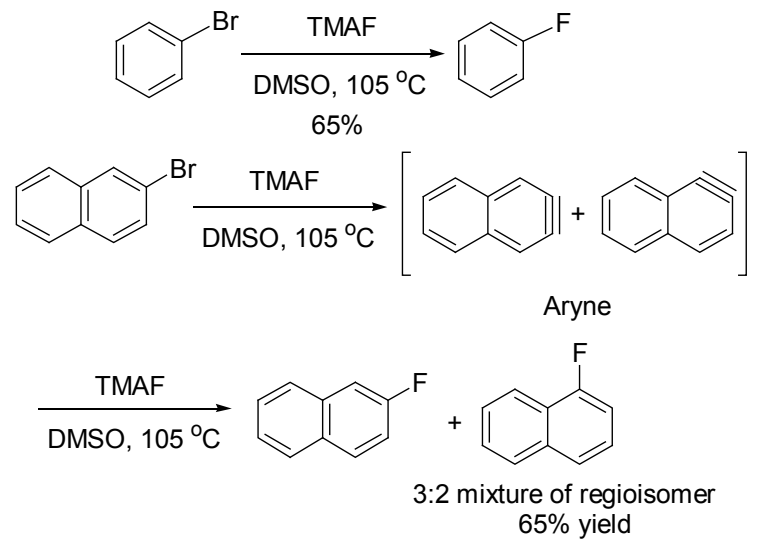

Scheme 17

\section{5 利用取代的苯酚和苯酚衍生物为原料}

从取代的苯酚和含羟基的取代的杂芳烃为起始原 料, Ritter 小组 ${ }^{[25]}$ 利用新颖的脱氧氟化试剂, 成功进行了 操作简易可行的酚类化合物的氟化(Eq. 5). 该转化具有 反应的产率高、基团兼容性好等优点.

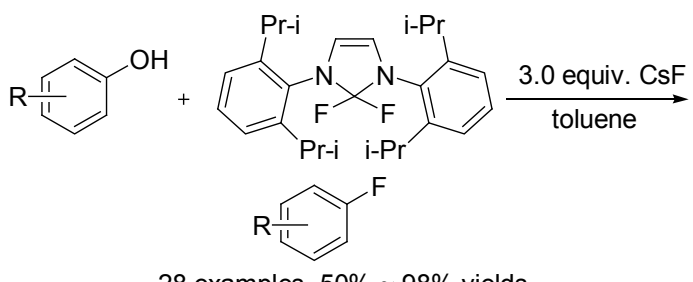

28 examples, $50 \% \sim 98 \%$ yields

经过芳基九氟丁磺酸酯中间体, Larhed 小组 ${ }^{[26]}$ 发现 了钯盐催化下的将酚类化合物转化为氟代芳烃的过程, 这个一个两步一锅法反应, 在微波辅助下完成, 收率良 好(Scheme 18).

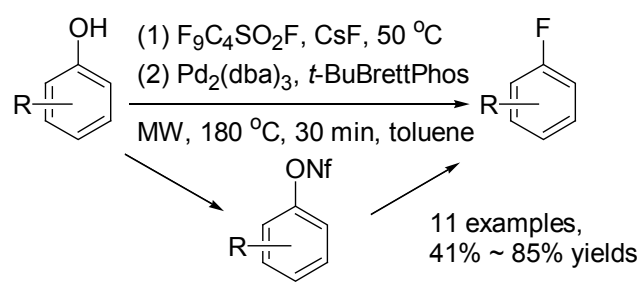

Scheme 18

2009 年 Buchwald 教授 ${ }^{[27]}$ 首先报道了以三氟甲磺酸 酯为原料的钯催化的亲核氟化反应，成功的关键是采用 了空间位阻大的特殊膦配体，并通过形成刚性 $\mathrm{T}$ 形的的 单核三配位的钯(II)络合物而完成反应. 有趣的是，在一 些反应中发现有区域异构体产生，产物以间位产物为主 (Scheme 19). 虽然目前对形成区域异构体的机理并不十 分清楚，但通过氟负离子进攻在原位的(in situ)产生的 苯炔活泼中间体的解释基本排除在外.
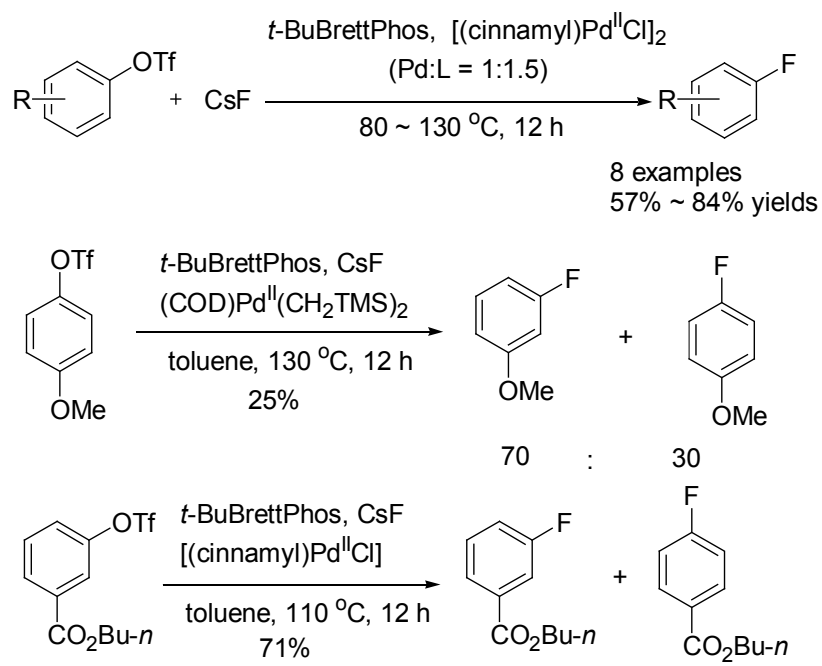

30

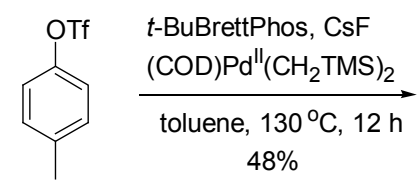

78

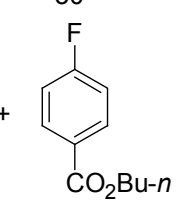

22

Scheme 19

钯催化的亲核氟化反应机理(Scheme 20)一般包括 三个步骤: (1)香卤代烃 $(X=B r, I)$ 或其三氟甲磺酸酯对 $\operatorname{Pd}(0)$ 的氧化加成; (2) $\mathrm{F}^{-}$参与的配体交换形成新的 $\operatorname{Pd}(\mathrm{II})$ 络合物; (3)通过还原消除形成 $\mathrm{C}-\mathrm{F}$ 键即产物.

由于邻苯二酚单元存在于许多重要的天然产物之 中，具有潜在的生物活性，对这些物质进行衍生化不失 为天然化合物改造的途径之一. 为此 Akai 小组 ${ }^{[28]}$ 利用 亲核脱氧氟化反应，通过在原位的(in situ)氧化, $\mathrm{F}^{-}$离子 加成和还原而制备了各种新颖的含氟类似物(Eq. 6). 


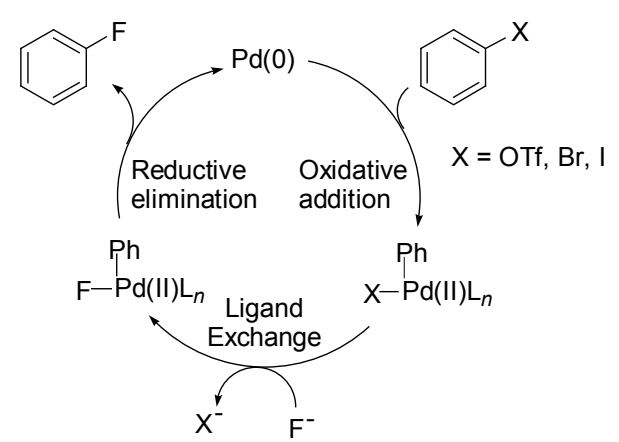

Scheme 20

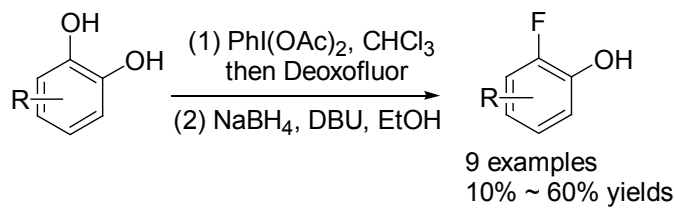

(6)

富电子的苯酚能和亲电的氟化试剂作用, 在羟基的 邻和对位上引入氟 ${ }^{[29]}$, 虽然该反应没有区域选择性, 但 也是一种进行氟扫描(Fluorine scan)的简易方法(Eq. 7). 所谓氟扫描, 就是通过化学合成的方法以母体化合物为 核, 制备一系列单氟化的衍生物, 也就是说芳(杂)环上 的各个位置上的 $\mathrm{C}-\mathrm{H}$ 键依次被 $\mathrm{C}-\mathrm{F}$ 键所取代, 而形成 新的含氟化合物. 这种方法逐渐被药物化学家接受和采 纳.<smiles>Oc1ccc(F)c(F)c1</smiles>

\section{6 利用芳香胺和酰替苯胺为原料}

芳香胺重氮盐的 $\mathrm{HF}$ /吡啶(Sandmeyer reaction)处理 和氟嗍酸处理(Balz-Schiemann reaction)是制备氟代芳烃 的老方法. 使用有机高价碘, Li 和 Meng 等 ${ }^{[30]}$ 成功进行 了免除金属的直接区域选择性氟化酰替苯胺衍生物, 将 氟原子引至酰替苯胺基的对位(Scheme 21). 该方法具有 操作安全、试剂易得、条件温和、产率较高等特点. 笔 者认为, 鉴于许多含氟药物都含有氟代苯胺结构单元 (见 Schemes 1 5), 在温和条件下芳香胺的选择性氟化 反应是值得认真研究的一个课题.

\section{7 利用芳基碘鎓盐和季铵盐为原料}

最近, Sanford 小组 ${ }^{[31]}$ 也成功研究出相转移条件下, 铜盐催化的不对称二芳基碘鎓盐的亲核氟化反应(Eq. 8). 该反应具有条件温和、反应快速、产率高、选择性 好、显示很好的基团兼容性以及没有位置异构体等优点.
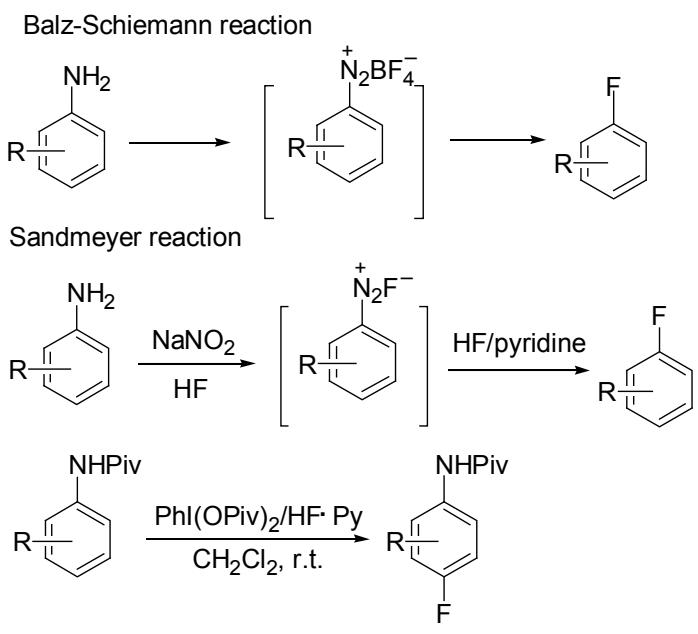

10 examples, $40 \% \sim 80 \%$ yields

\section{Scheme 21}

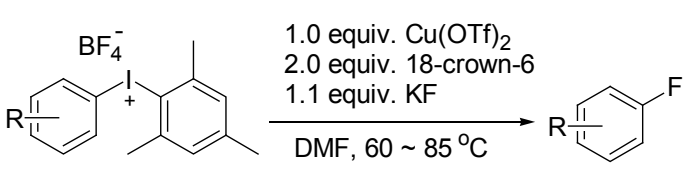

26 examples, $54 \% \sim 98 \%$ yields

除了芳基碘鎓盐外，季铵盐也不失为合理的离去基 才，据报道在非常温和的条件下，2,3,5,6-四氟苯基尼古 丁酸酯的季铵盐能够有效地进行 ${ }^{18} \mathrm{~F}$ 的同位素标记 ${ }^{[32]}$ (Eq. 9), 由于这是一个免除过渡金属钯及铜盐的过程, 操作使用及后处理方便, 其在临床医学上应用的前景具 有诸多优点.

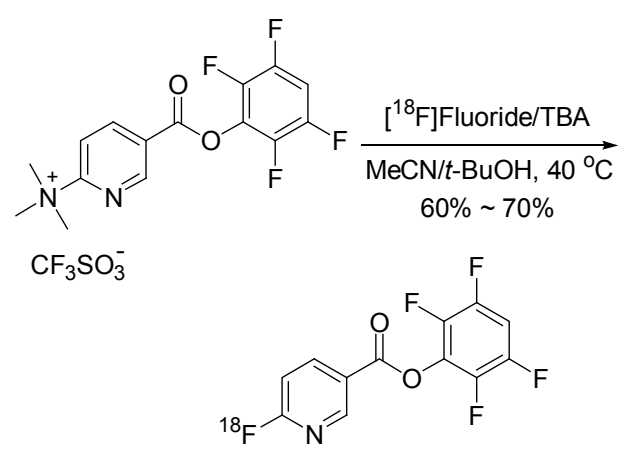

\section{8 后氟化(Late-stage fluorination)反应制备药物和 天然产物的氟代衍生物}

Ritter 小组 ${ }^{[19,20]}$ 利用他们率先发现的银盐催化的后 氟化(Late-stage fluorination)反应策略, 成功制备了数十 个重要的上市药物和天然产物的氟代衍生物, 其中包括 氟代 Taxol、氟代 DOPA、氟代 Rifamycin $S$ 和氟代 Camptothecin 等(Scheme 22). 对于代谢不稳定的碳-氢 $(\mathrm{C}-\mathrm{H})$ 位点可以采用碳-氟 $(\mathrm{C}-\mathrm{F})$ 键进行取代, 这也许 能够有效地改善药物的药代动力学特性. 这一工作对药 物化学工作者在新药笁选和优化中是有启示作用的. 当 
然对我国药物化学工作者进行新药改造也会有帮助的.
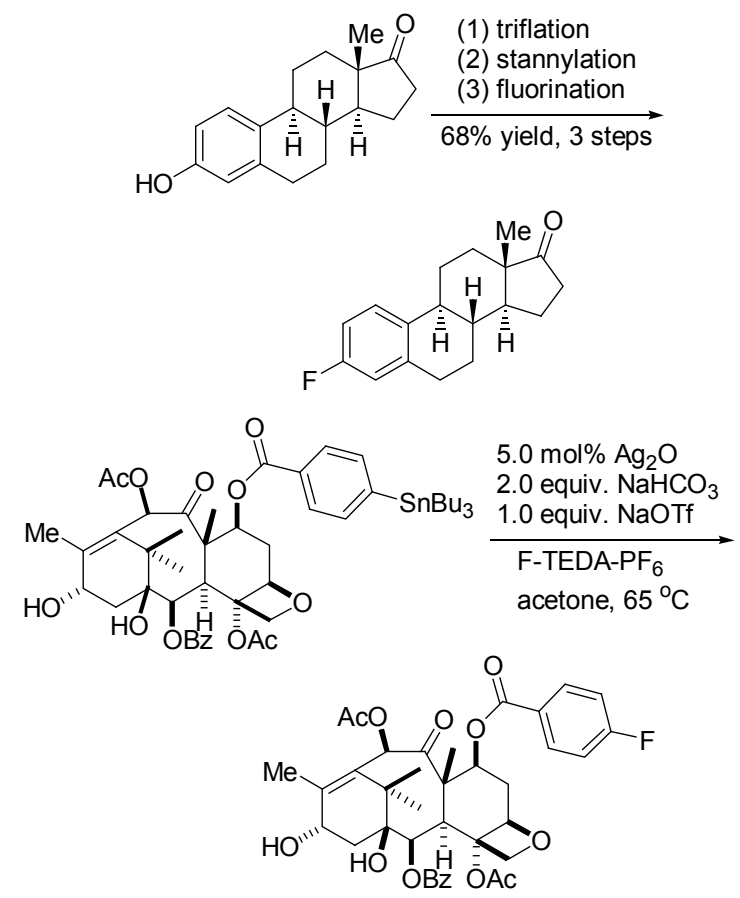

Scheme 22

\section{2 芳烃和杂芳烃二氟甲基化反应}

胡金波等 ${ }^{[33]}$ 于 2009 年已将选择性二氟甲基化和单 氟甲基化做了很好的综述. 相对于芳烃的三氟甲基化和 芳香烃的氟化反应，芳烃和杂芳烃的二氟甲基化研究略 显薄弱, 方法学方面的研究也显零散. 但这一状况最近 得到了很大的改观. 在这里我们对近年的新进展做一概 括.

虽然迄今为止，仍然没有上市的药物中含有二氟甲 基 $\left(\mathrm{CHF}_{2}\right)$ 芳杂环亚结构单元的 $\mathrm{APl}$, 但是二氟甲基独特 的结构特点仍然吸引了许多药物化学家的注意. 在等排 物为基础的药物设计中, $\mathrm{CHF}_{2}$ 不失为优秀的亲脂性的氢 键供应者, 为传统的氢键供应源提供了新的选择, 并且 同时能有效地改善药物的膜渗透性，促进药物的吸收. 例如在抗丙肝病毒(HCV NS3)蛋白酶抑制剂的研发过程 中 ${ }^{[34]}$ ，成功地利用二氟甲基取代并模仿母体化合物中硫 醇的功能. 在 COX-2 和 5-LOX 双重抑制剂的研发过程 中 $^{[35]}$ ，二氟甲基可做为异羟肜酸中羟基的等排物.

传统的二氟甲基 $\left(\mathrm{CF}_{2} \mathrm{H}\right)$ 的引入一般都是由芳香醛 和 DAST 试剂的反应而完成的. 考虑到有机磺酸盐中 $\mathrm{C}-\mathrm{S}$ 键 $(272 \mathrm{~kJ} / \mathrm{mol})$ 比有机嗍酸 $\mathrm{C}-\mathrm{B}(377 \mathrm{~kJ} / \mathrm{mol})$ 键和 有机分子中的 $\mathrm{C}-\mathrm{C} 348 \mathrm{~kJ} / \mathrm{mol}$ )要弱许多, Scripps 的 Baran 小组 ${ }^{[36]}$ 成功制备了含二氟甲基结构的亚磺酸锌盐 (DFMS), 利用过氧化叔丁醇为引发剂, 有效地均裂 C$\mathrm{S}$ 键, 成功地制备了 $\mathrm{CF}_{2} \mathrm{H}$ 游离基, 并成功开发了相关反
应(Scheme 23).

$$
\begin{aligned}
& \mathrm{CHF}_{2} \mathrm{SO}_{2} \mathrm{Cl} \underset{\mathrm{H}_{2} \mathrm{O}}{\mathrm{Zn}}\left(\mathrm{CHF}_{2} \mathrm{SO}_{2}\right)_{2} \mathrm{Zn} \\
& \stackrel{\text { Het }}{-} \mathrm{H}+\left(\mathrm{CHF}_{2} \mathrm{SO}_{2}\right)_{2} \mathrm{Zn} \underset{\mathrm{CH}_{2} \mathrm{Cl}_{2}, \mathrm{H}_{2} \mathrm{O}}{\stackrel{t}{\mathrm{H}} \mathrm{BuOt}}-\mathrm{CF}_{2} \mathrm{H} \\
& 20 \text { examples, } 30 \% \sim 90 \% \text { yields }
\end{aligned}
$$

Scheme 23

反应具有下面一些特点 ${ }^{[37]}:$ (1) DFMS 试剂稳定, 不 怕水不怕氧；(2)反应可在敞开体系进行; (3)反应在室温 下进行，水可作为溶剂之一; (4)反应展示良好的区域选 择性; (5)缺电子和富电子的杂环也能参与反应(Eq. 10); (6)卤素原子不干扰反应; (7)操作简单易行，该试剂已商 业化.

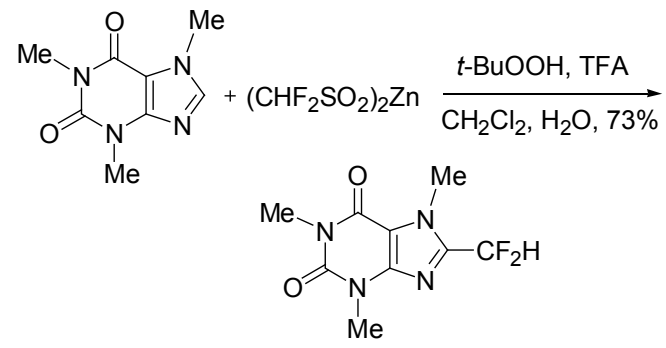

值得注意的是，Baran 小组对一些天然产物和上市 的重磅炸弹药物, 如辉瑞制药的 Chantix 进行了后三氟 甲基化(Late-stage trifluoromethylation)和后二氟甲基化 (Late-stage difluoromethylation)过程，在不同的反应条件 下，经过不同的反应机理，在母体化合物上有效地引进 了 $\mathrm{CF}_{3}$ 和 $\mathrm{CF}_{2} \mathrm{H}$ 官能团(Scheme 24), 这不失为药物创新 改造和开拓构效关系(SAR)研究的新方法和快速通道. 从某种意义来说，后 $\mathrm{N}(n=2,3)$ 氟甲基化也是将芳(杂) 环电子云密度大或着是电子云密度小的 $\mathrm{C}-\mathrm{H}$ 键(即反 应的活性位点)通过亲电取代和亲核取代这两种截然不 同的反应被 $\mathrm{C}-\mathrm{CF}_{3}$ (或 $\mathrm{C}-\mathrm{CHF}_{2}$ )健取代，应该是阻止药 物氧化代谢的极好手段，也是提高药物生物利用度的方 便策略.

最近, Chen 小组 ${ }^{[38]}$ 报道了可见光下无金属(Metalfree)的 C- $\mathrm{H}$ 活化过程，巧妙地使用 $5 \mathrm{~mol} \%$ 氧杂蒽酮做 为茮基氢的捕获者，成功进行了光催化循环，在非常温 和的条件下制备了一系列带有二氟甲基化支键的芳香 烃(Eq. 11). 反应具有操作简单方便等优点, 不失为一种 绿色的二氟甲基化新手段.

利用碘苯和 2-三乙基硅-2，2-二氟代乙酸乙酯的交 叉偶联反应制备芳香基取代的二氟代乙酸酯，并伴随水 解和脱羧, Amii 小组 ${ }^{[39]}$ 用两步法完成了芳香环的二氟甲 基化(Scheme 25). 比较陈庆云院士上世纪九十年代的方 法，发现该法仍有脱羧反应较难的缺点 $\left(170 \sim 200{ }^{\circ} \mathrm{C}\right)$. 

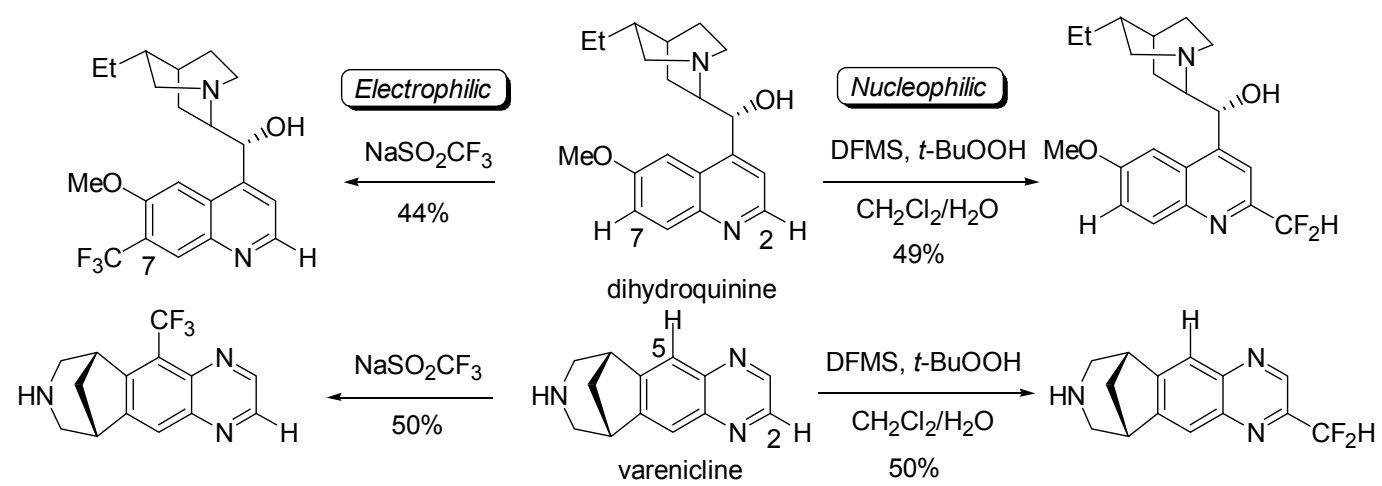

Scheme 24

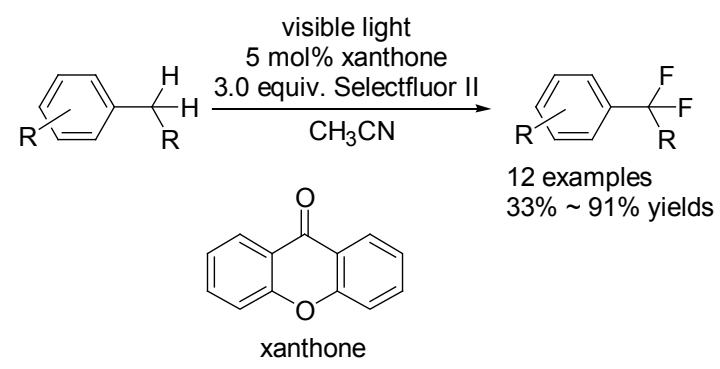

$$
+\mathrm{Me}_{3} \mathrm{SiCF}_{2} \mathrm{COOEt} \frac{1.2 \text { equiv. Cul }}{\mathrm{DMSO}, 60^{\circ} \mathrm{C}}
$$$$
\begin{aligned}
11 \text { examples } \\
41 \% \sim 88 \% \text { yields }
\end{aligned}
$$

\section{Scheme 25}

将著名的 Ruppert-Prakash 试剂( $\left.\mathrm{TMSCF}_{3}\right)$ 经过硼氢 化钠还原, Hartwig 小组 ${ }^{[40]}$ 以 $70 \%$ 的收率制备了新的二 氟甲基化试剂 $\mathrm{TMSCF}_{2} \mathrm{H}$, 并成功应用于铜盐催化的二 氟甲基化碘苯(Eq. 12), 反应的收率高, 操作简单易行, 对各种官能团具有良好的兼容性.

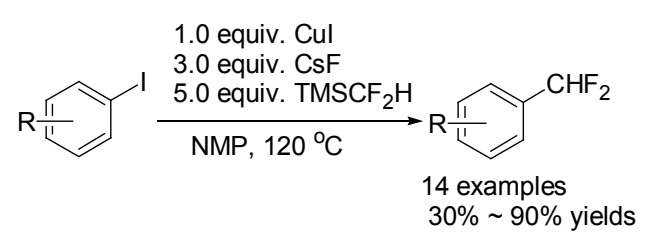

\section{3 芳(杂)环的氟甲基化反应}

类似于三氟甲基 $\left(\mathrm{CF}_{3}\right)$ 、单氟甲基 $\left(\mathrm{CH}_{2} \mathrm{~F}\right)$ 可以被认为 是甲基的有用的生物电子等排体. 其原因是氟的强吸电 子效应使得氟原子能避免甲基的代谢性氧化. 而且单氟 甲基也被认为是羟甲基 $\left(\mathrm{CH}_{2} \mathrm{OH}\right)$ 和甲氧基甲基 $\left(\mathrm{CH}_{2} \mathrm{OCH}_{3}\right)$ 的生物电子等排体. 据调查, 真正意义上的 单氟甲基化方法几乎不存在, 尤其是通过产生单氟甲基 游离基. 应该承认单氟甲基化是难度很大、富有挑战的

研究热点, 但这方面的工作最近取得了很大的进展, 其 中包括 Scripps 的 Baran 教授的新试剂 $\left(\mathrm{CH}_{2} \mathrm{FSO}_{2}\right)_{2} \mathrm{Zn}$ 及 方法 ${ }^{[37]}$ (Scheme 26).

$$
\begin{aligned}
& \mathrm{CH}_{2} \mathrm{FSO}_{2} \mathrm{Cl} \underset{\mathrm{H}_{2} \mathrm{O}}{\mathrm{Zn}}\left(\mathrm{CH}_{2} \mathrm{FSO}_{2}\right)_{2} \mathrm{Zn} \\
& \text { Het } \mathrm{H}+\left(\mathrm{CH}_{2} \mathrm{FSO}_{2}\right)_{2} \mathrm{Zn} \frac{t \text {-BuOOH, TFA }}{\mathrm{CH}_{2} \mathrm{Cl}_{2}, \mathrm{H}_{2} \mathrm{O}} \mathrm{CH}_{2} \mathrm{~F} \\
& 5 \text { examples } \\
& 40 \% \sim 80 \% \text { yields (GC) }
\end{aligned}
$$

Scheme 26

在钯盐催化和 $\mathrm{AgF}$ 的作用下，一些杂芳环如含甲基 取代的喹啉化合物也能进行茮基氟化反应(Eq. 13) ${ }^{[41]}$, 虽然反应的收率仍有待提高.
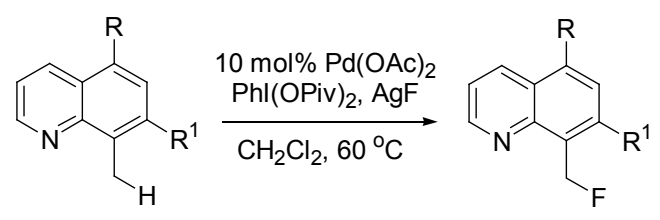

10 examples $30 \% \sim 70 \%$ yields

使用活性的芳香酮如 9-芴酮作为可见光下茮基氢 的捕食物, Chen 小组 ${ }^{[38]}$ 首次报告了选择性产生苠基游离 基并通过加成氟游离基而完成苠基氟化的催化循环(Eq. 14). 值得一提的是通过巧用、活用不同的芳香酮作为光 催化剂, 有效方便地制备了多达数十个结构各异、官能 团兼容的带有氟甲基支键的芳香烃. 应该指出的是这也 是迄今为止茮基氟化反应的最新进展.

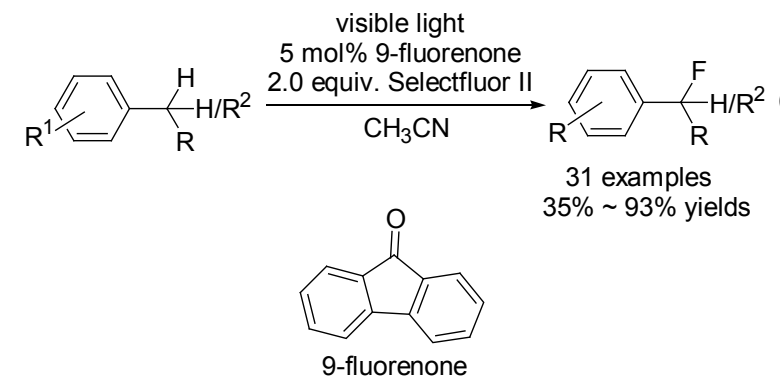




\section{4 三氟甲基化反应亮点}

传承优秀的中国氟化学研究团队数十年来科研成 果突出, 硕果累累, 亮点不断, 一直深受国际学术界的 赞叹, 最近中国科学院上海有机化学所的卿风领教授 ${ }^{[5]}$ 已经将近年来(2009 2011 年)三氟甲基化的研究进展做 了较为深入的总结和归纳. 北京大学王剑波教授 ${ }^{[2]}$ 最近 也对经由三氟甲基自由基进行的三氟甲基化的一些基 本理论问题做了很好的探讨, 而西班牙的氟化学专家 Grushin 也对金属参与的芳烃三氟甲基化在 Chem. Rev 上发表了系统全面的综述 ${ }^{[43]}$. 考虑到对三氟甲基化反应 研究方兴未艾 ${ }^{[44]}$, 这方面的高水平研究论文层出不穷, 我们仅将近年以来三氟甲基化研究的亮点从简易方便 即实用性, 高效催化即绿色性和安全放大即工艺合理性 等角度做一点评, 挂一漏万有偏颇之处, 在所难免.

近年来, 不少新型的三氟甲基化试剂, 如正离子型 的、负离子型(Scheme 27)的和游离基型的 $\mathrm{CF}_{3}$ 来源, 它 们为进行亲电、亲核和游离基三氟甲基化提供了选择的 可能，并成为反应条件优化必须考虑的主要因素.

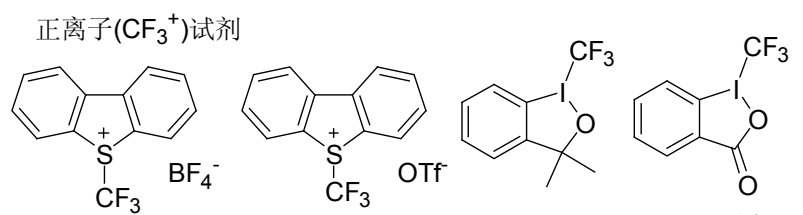<smiles>O=S(=O)(c1ccccc1)C(F)(F)[Mg]Br</smiles>

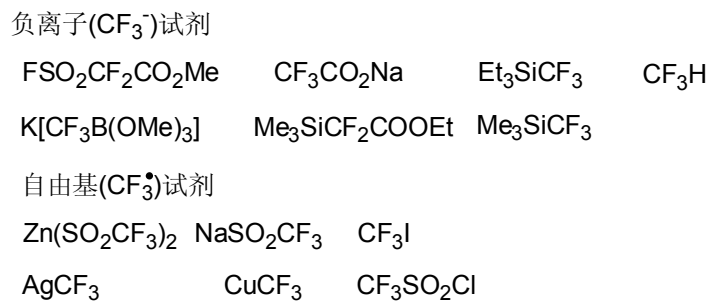

Scheme 27

过渡金属催化的三氟甲基化反应在有机氟化学家 和金属有机化学家的联手努力下，已经取得了突破性的 进展(Eq. 15). 但不可否认, 和成熟的钯催化的交叉偶联 反应(Suzuki, Heck, Negishi)相比较, 改善的空间仍然是 巨大的, 其主要缺陷是一般情况下钯催化剂的用量较高 (10\%)，三氟甲基化试剂一般较为昂贵，而且有些试剂 为气体或低沸点小分子, 物理化学性质不理想也是大气 臭氧层的破坏者, 再者, 过渡金属的含量在药物分子 API 中的控制也是必须考虑的因素之一. 从这个方面来 讲, Scripps 的 Baran 小组 ${ }^{[45]}$ 在 Langlosi 小组 ${ }^{[46]}$ 前期工作
基础上将便宜易得的三氟亚磺酸钠作为三氟甲基源，过 氧叔丁醇作为氧化剂而进行的杂环的直接三氟甲基化. 由于反应不需要金属催化剂, 能在室温下进行, 反应条 件温和，溶剂为水/乙腈(也不需额外处理)，对各种取代 基及官能团不需保护和耐受性好，是一种实用价值很强 且易放大和工业化的好方法，堪称一大突破. 当然对自 由基参与的化学反应，反应热的控制和安全性评价是必 不可少的.

$$
\text { Het } \frac{\mathrm{NaSO}_{2} \mathrm{CF}_{3}(3.0 \sim 6.0 \text { equiv. })}{t-\mathrm{BuOOH}(5.0 \sim 10.0 \text { equiv. })}
$$

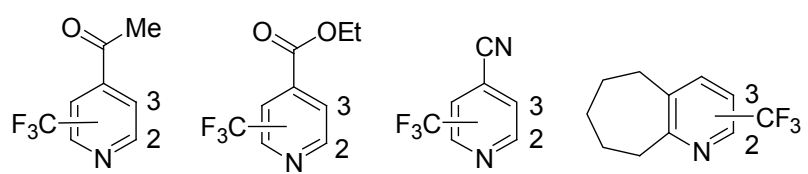<smiles>CC(=O)c1ccc(C(F)(F)F)[nH]1</smiles><smiles>Nc1nnc(C(F)(F)F)s1</smiles><smiles>COC(=O)c1cnc(C(F)(F)F)cn1</smiles><smiles>CCc1nc(CC)c(C(F)(F)F)nc1C(F)(F)F</smiles><smiles>Cc1nc2ccccc2nc1N</smiles><smiles>O=c1[nH]cc(C(F)(F)F)c(=O)[nH]1</smiles><smiles>FC(F)(F)c1c[nH]c2ncnc(Cl)c12</smiles>

有趣的是，该三氟甲基化反应是通过自由基中间体 完成的，因此反应的区域选择性并不是高度专一的，从 某种程度来说，它对现存药物的三氟甲基化而带来的新 的药物分子的多样性确是一件好事，因为芳烃或杂环分 子有多于一个的活性反应点, 而便利地引入三氟甲基改 造现有药物分子不失为一种旧药改造的捷径. Scheme 28 列出了一系列经过后三氟甲基化 (Late-stage trifluoromethylation)的天然产物和重磅炸弹，我们相信 许多药物研发的科学家也会逐渐学习接纳这一新手段.

由于芳香硼酸的来源广泛和易制备，近年来，采用 $\mathrm{CF}_{3}^{+}$的铜媒促的三氟甲基化反应取得了很大的进展, 但诸多新方法均有采用昂贵的试剂如 $\mathrm{TMSCF}_{3}$, Togni's 和 Umemoto's 试剂, 以及严格的实验条件, 如需要惰性 气体保护和干燥的溶剂等限制，不利于大规模制备. 鉴 于此, 2012 年 Sanford 小组 ${ }^{[47]}$ 先后报道了 $\mathrm{CF}_{3} \mathrm{I}$ 和 
<smiles>COc1ccc2[nH]c(C(F)(F)F)c(CCNC(=O)C(F)(F)F)c2c1</smiles>

Scheme 28

$\mathrm{CF}_{3} \mathrm{SO}_{2} \mathrm{Na}$ 在铜盐催化下经由 $\mathrm{CF}_{3}$ 游离基参与的三氟甲 基化反应(Eq. 16), 避开了使用气态的 $\mathrm{CF}_{3} \mathrm{I}$ 和昂贵的 $\mathrm{Ru}$ 催化剂, 仅使用便宜易得的 $\mathrm{CF}_{3} \mathrm{SO}_{2} \mathrm{Na}$ 试剂, 并在过氧 化叔丁醇( TBHP)的引发下, 使用 $\mathrm{CuCl}$ 或其它试剂, 在 非常温和的条件下(室温下, 空气中, 不需纯化试剂和溶 剂)顺利地完成了转化. 产率高达 96\%.

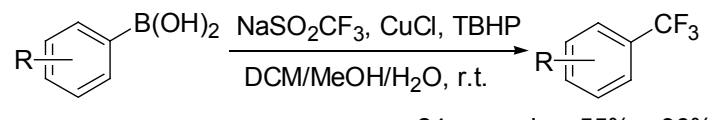

24 examples, $55 \% \sim 96 \%$ yields

2012 年 Grushin 研究小组 ${ }^{[48]}$ 也报道了利用氟仿(氟 利昂)制备 $\mathrm{CuCF}_{3}$ 试剂在空气中进行的芳香嗍酸参与的 三氟甲基化反应(Scheme 29), 在室温和空气存在下高产 率地制备了芳香三氟甲基化合物. 据认为反应机理是将 一价的具有亲核的 $\mathrm{Cu}(\mathrm{I}) \mathrm{CF}_{3}$ 在空气中转化为二价且具 有亲电的 $\mathrm{Cu}(\mathrm{II}) \mathrm{CF}_{3}$, 并通过金属交换和还原消除而实 现的. 美中不足的是氟利昂是气体(b.p. $\left.-84{ }^{\circ} \mathrm{C}\right)$, 也是 产生温室效应而受到控制的有害物质之一.

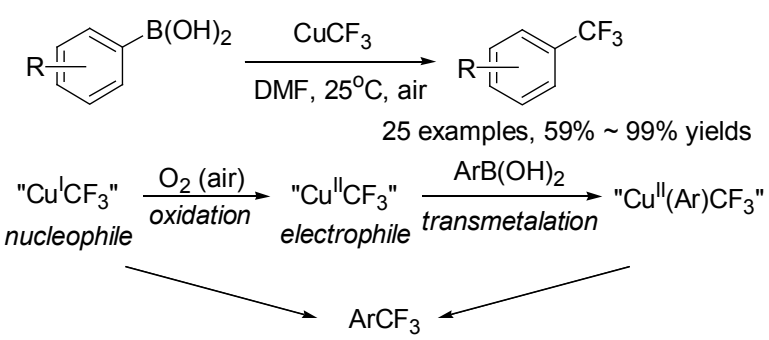

\section{Scheme 29}

利用桑德迈尔(Sandmeyer)这一经典的反应进行三 氟甲基化芳烃是中国化学工作者在 2013 年对有机氟化
学反应的一大贡献. 中国科技大学的傅尧和其研究团 队 ${ }^{[49]}$ (包括清华大学的刘磊等)首先报道了铜盐媒促下, 使用 Umemoto 试剂在极其温和的条件下 $\left(0 \sim 15{ }^{\circ} \mathrm{C}\right)$ 以 芳香胺为原料，经过重氮盐中间体完成的三氟甲基化反 应(Eq. 17). 反应具有操作简单、原料易得、底物变化多 样、反应物中的多种官能团并不需要保护处理、兼容性 好以及高度的区域选择性等特点, 不失为一种应用前景 广泛的从胺基到三氟甲基的基团转换.

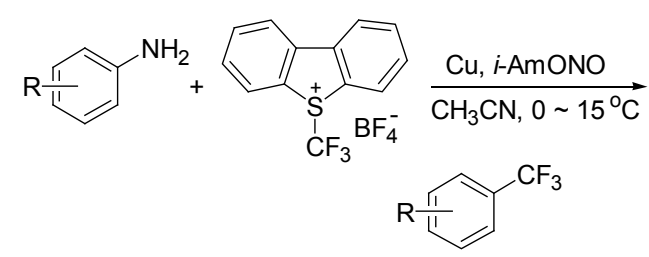

19 examples, 41 89\% yields

对反应机理的研究表明，这个一个铜促产生单电子 转移(SET)而形成 $\mathrm{CuCF}_{3}$ 试剂, 并和芳基游离基作用而 形成最终产物的过程(Scheme 30).

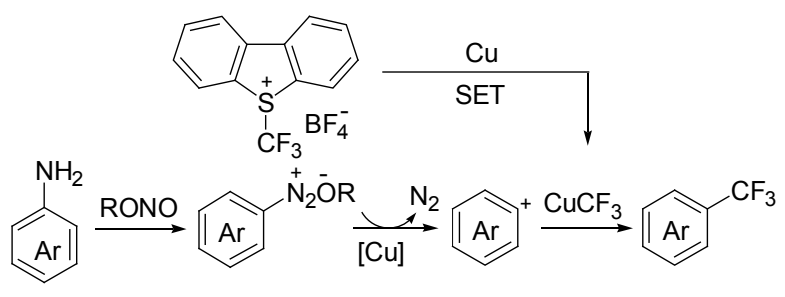

\section{Scheme 30}

Gooßen 研究小组 ${ }^{[50]}$ 随后报道了铜盐催化下, 使用 Ruppert 试剂 $\left(\mathrm{TMSCF}_{3}\right)$ 以芳香胺为原料的桑徳迈尔反应 制备三氟甲基化芳烃(Scheme 31). 有所不同的是，他们 使用的催化剂是一价铜盐, 其中以硫氧化铜 $(\mathrm{CuSCN})$ 催 化活性最高. 反应温度为室温, 有很好的官能团兼容性, 产率在 $40 \% \sim 98 \%$ 之间.

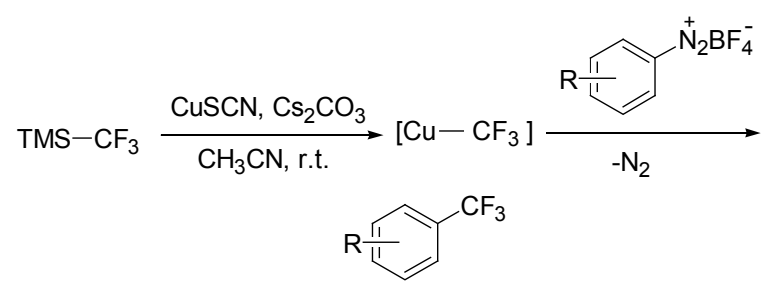

19 examples $40 \% \sim 98 \%$ yields

\section{Scheme 31}

王剑波等 ${ }^{[51]}$ 首先报道了通过三氟甲基银 $\left(\mathrm{AgCF}_{3}\right)$ 和 芳香胺参与的桑德迈尔反应来完成胺基至三氟甲基的 官能团转化(Scheme 32). 值得一提的是, 反应需要在一 $78{ }^{\circ} \mathrm{C}$ 下完成, 否则收率较低. 三氟甲基银是通过 $\mathrm{AgF}$ 
和 $\mathrm{TMSCF}_{3}$ 来制备的, 有趣的是: 使用 $\mathrm{CuCF}_{3}$ 为试剂, 在相似条件下, 产物的收率不佳 $(37 \%)$. 避免使用超低 温反应条件 $\left(-78{ }^{\circ} \mathrm{C}\right)$ 也许是将来反应优化、走向适用性 的发展方向之一.

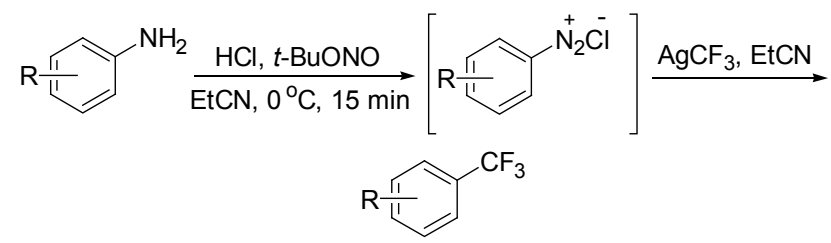

48 examples, $10 \% \sim 95 \%$ yields

\section{Scheme 32}

傅尧小组 ${ }^{[49]}$ 使用他们首先发现的芳香胺参与的桑 德迈尔三氟甲基化重氮盐反应，经过两步反应成功制备 了抗风湿关节炎药物 Leflunomide. 与此同时, 他们也采 用后三氟甲基化(Late-stage trifluoromethylation)策略合 成抗老年痴呆药物 Aricept 的关键三氟甲基衍生化中间 体(Scheme 33). 应该指出的是, 现存上市药物的三氟甲 基衍生化不失为发掘老药新用及改造的捷径之一, 值得 我国的药物化学工作者好好利用.<smiles>CCN(CC)CCc1ccccc1C(=O)Nc1ccc(N)cc1</smiles><smiles>CC(=O)c1ccc(NC(=O)c2cnoc2C)cc1</smiles><smiles>COc1cc2c(cc1OC)C(=O)[C@H]([C@H](C)CCCCO)C2</smiles><smiles>COc1cc2c(c(N)c1OC)C(=O)CCC21CCCC1</smiles><smiles>COc1cc2c(c(C(F)(F)F)c1OC)C(=O)CC2CC1CCN(Cc2ccccc2)CC1</smiles>

Scheme 33
Baran 研究小组 ${ }^{[45]}$ 在利用便宜易得的三氟亚磺酸钠 进行芳烃三氟甲基化工作的基础上，最近又成功地从三 氟亚磺酰氯和锌粉在水为溶剂中制备了物化性能更加 优越的新试剂三氟亚磺酸锌, 并成功应用于芳香烃的三 氟甲基化反应(Scheme 34). 利用该试剂, Baran 研究小组 对一些天然产物，药物中间体也进行了后三氟甲基化 (Late stage trifluoromethylation), 并取得了理想的实验结 果.

$$
\begin{aligned}
& \mathrm{CF}_{3} \mathrm{SO}_{2} \mathrm{Cl} \underset{\mathrm{H}_{2} \mathrm{O}}{\stackrel{\mathrm{Zn}}{\longrightarrow}}\left(\mathrm{CF}_{3} \mathrm{SO}_{2}\right)_{2} \mathrm{Zn}
\end{aligned}
$$

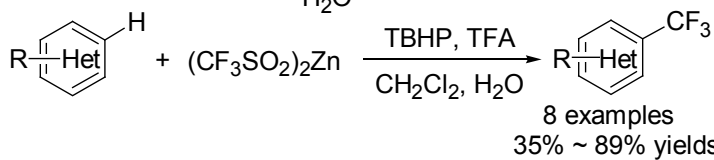

Scheme 34

使用三氟亚磺酰氯, 在 $\mathrm{Ru}(\mathrm{phen})_{3} \mathrm{Cl}_{2}$ 光催化剂存在 下, 普林斯顿大学的 MacMillan 小组 ${ }^{[52]}$ 成功对数个上市 药物, 包括 Lipitor 和 Ibuprofen 进行了后三氟甲基化作 用(Eq. 18), 该方法也具有条件温和、产率高、操作方便 等特点.
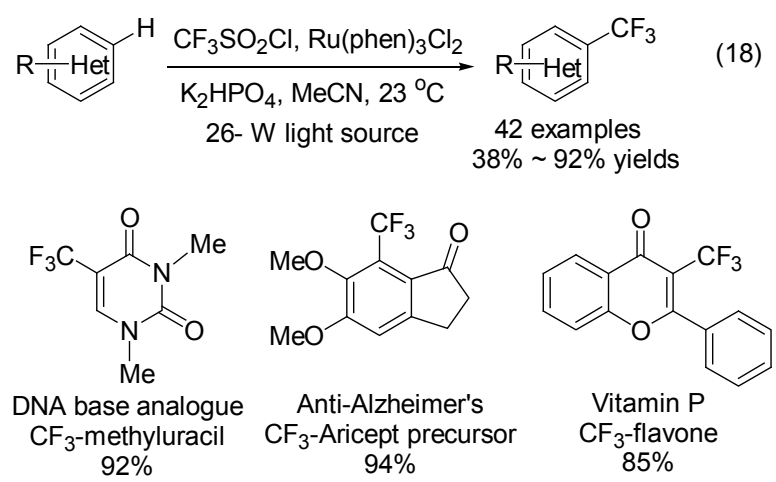

芳杂环尤其是含氮芳杂环是药物研发中常常使用 的优选结构单元, 许多含杂环的候选化合物常常有溶解 度不佳、吸收和代谢困难等诸多缺陷. 在杂环上选择性 地引入三氟甲基，能够有效地改变药物的物理化学性 质，例如 Merck 的糖尿病新药 Sitagliptin 中就含有关键 的三氟甲基化的三唑基团. 值得一提的是在杂芳环的三 氟甲基化研究方面, 中国科学院上海有机化学研究所的 数个研究课题组取得了许多令人瞩目的突破. 采用直接 的 $\mathrm{CH}$ 活化策略, 卿风领小组 ${ }^{[53]}$ 首先发现了在醋酸铜催 化/配体和双碱 $t-\mathrm{BuONa} / \mathrm{NaOAc}$ 的存在下，使用空气或 过氧化叔丁基醚为氧化剂、经由 $\mathrm{CF}_{3} \mathrm{TMS}$ 作用完成的氧 化三氟甲基化(oxidative trifluoromethylation)噁二唑反应 (Eq. 19). 该转化具有产率高, 反应条件温和等优点，应 该成为候选药物分子后三氟甲基化的好方法. 值得一起 的是在芳环一杂环共存的条件下，反应具有优越的区域 选择性. 


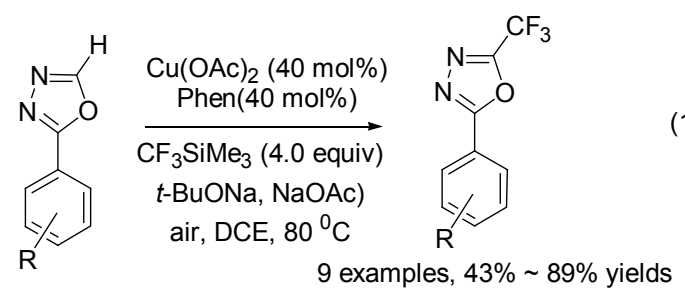

卿凤翎的上述新颖方法也成功地扩展到苯并噁唑、 苯并咪唑、苯并噻唑和取代吲哚等杂环系统以及缺电子 的全氟芳烃的三氟甲基化, 均取得了很好的实验结果 (Eq. 20).

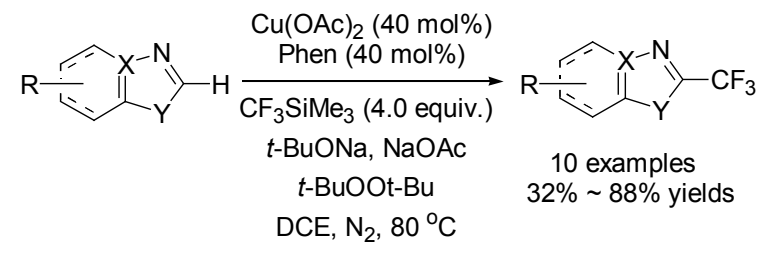<smiles>FC(F)(F)c1nc2cc(-c3ccccc3)ccc2o1</smiles>

$74 \%$<smiles>Cn1c(C(F)(F)C(F)(F)F)nc2ccccc21</smiles>

$57 \%$<smiles>Cc1ccc2oc(C(F)(F)F)nc2c1</smiles>

$72 \%$
2012 年沈其龙小组 ${ }^{[54]}$ 发展了一个 $\mathrm{Ir} / \mathrm{Cu}$ 协同催化的 一锅法 $\mathrm{C}-\mathrm{H}$ 键活化 $\mathrm{C}-\mathrm{B}$ 键生成、三氟甲基化新反应 (Eq. 21). 上述催化方法也成功地应用到 2,6-二取代吡 啶、苯并呋喃、苯并噻唑、取代吲哚和取代喹啉等杂环 系统, 均取得了良好的实验结果. 该方法的优点是可以 对有机药物在发展后期定点选择性地活化碳氢键并引 入三氟甲基, 简化合成步骤, 加快寻找药物的先导化合 物.
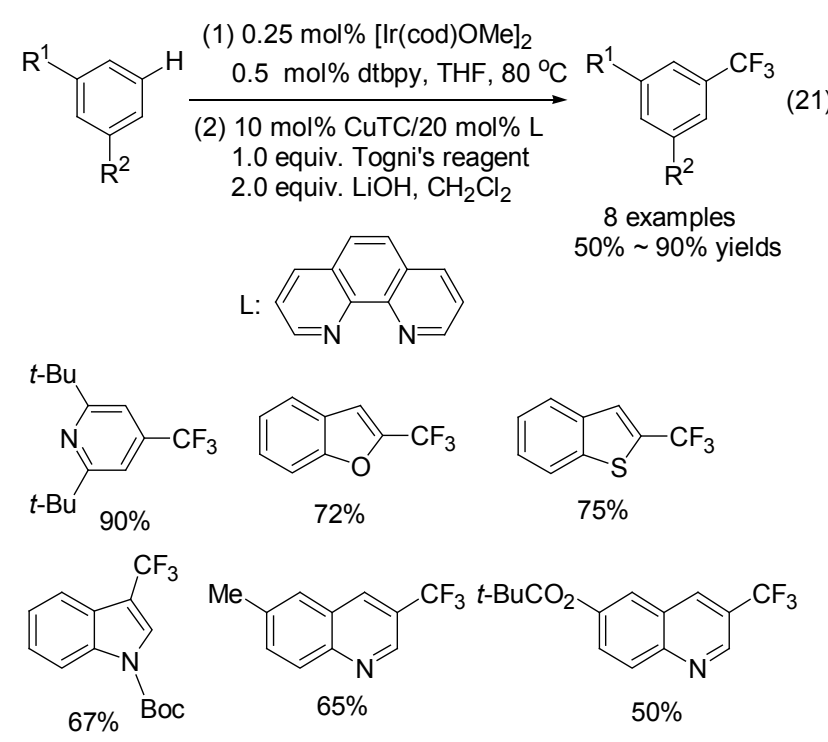

肖吉昌小组 ${ }^{[55]}$ 也首次报道了铜促的采用三氟甲基 锍盐的三氟甲基化碘代芳杂(稠)环的新方法(Eq. 22). 该 反应具有官能团兼容性好、反应条件温和、产率高等优 点，对不同杂环系统的三氟甲基化均取得了很好的结 果. 据称反应机理涉及到铜还原锍盐产生活泼中间体 $\mathrm{CuCF}_{3}$ 的单电子转移过程.

$$
\text { 药 }
$$

使用 $10 \mathrm{~mol} \%$ 醋酸钯为催化剂、 $\mathrm{TMSCF}_{3}$ 为三氟甲 基化试剂, $\mathrm{L}$ 为配体、 $\mathrm{PhI}(\mathrm{OAc})_{2}$ 为氧化剂, 刘国生小 组 ${ }^{[56]}$ 成功完成了室温条件下的 $\mathrm{N}$-甲基吲哚的三氟甲基 化(Eq. 23).

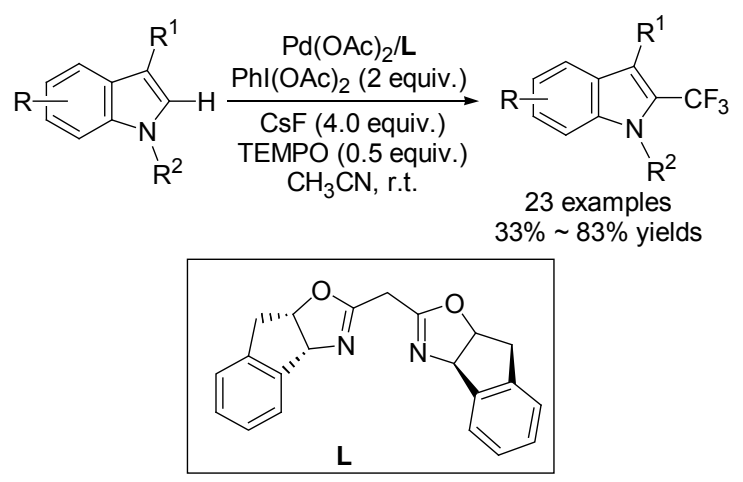

最近，曹松等 ${ }^{[57]}$ 对取代三唑的三氟甲基化也进行了 很好的研究, 他们发现以取代芳烃 5-碘代三唑为原料, 在 $\mathrm{Cul} / \mathrm{KF} / \mathrm{Ag}_{2} \mathrm{CO}_{3}$ 和配体共存条件下，能够极为有效地 引入三氟甲基(Eq. 24). 鉴于取代三唑为很好的药效基 (尤其是一系列沙坦类药物中广泛存在), 这一便利方法 相信会得到药物工作者的喜欢.

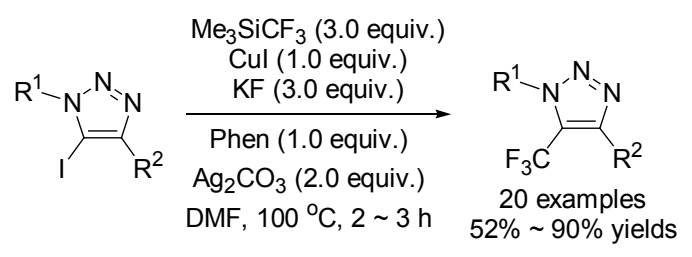

2013 年初, 胡金波小组 ${ }^{[58]}$ 首先报道了新颖别致的 同时在芳烃上引入三氟甲基和碘的新反应(Eq. 25). 这 是一个银盐媒促的、通过苯炔中间体一步生成邻位两个 重要取代基的新手段. 他们对三氟甲基的来源、亲电试 剂、配体(TMP $=2,2,6,6$-tetramethylpiperidine)等诸多因 素进行了笛选, 成功地发现了这个新方法. 值得一提的 是碘代的三氟甲基苯环是进行金属催化偶联的关键中 间体，应该成为理想的合成原料，同时反应的副产物之 一为炔银, 从原子经济学的角度来说, 仍然有进一步利 
用的空间和可能.

$$
\text { 等 }
$$

值得一提的是, 2010 年 $\mathrm{Yu}$ 等 ${ }^{[59]}$ 利用一系列杂环如 吡啶、嘧啶、咪唑和噻唑为邻位导向基, 在 $\mathrm{Pd}(\mathrm{OAc})_{2}$ 的 催化和 TFA 为促进剂的联合作用下, 成功地利用 $\mathrm{C}-\mathrm{H}$ 活化方法进行了芳环的三氟甲基化, 取得了良好的实验 结果(Eq. 26).

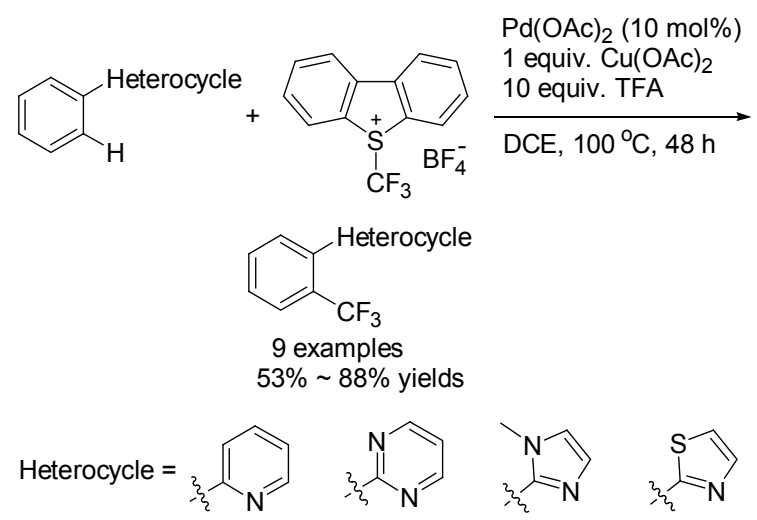

\section{5 氟化反应的放大和安全使用}

20 世纪 80 年代至 90 年代初, 陈庆云院士领导的研 究团队 ${ }^{[60]}$ 先后发现了数个三氟甲基化的试剂 ${ }^{[5]}$, 其中 1991 年报道的 1,1-二氟-1-氯代乙酸甲酯(MCDFA) 在 KF 和 $\mathrm{CuI}$ 存在下在 $\mathrm{DMF}$ 溶剂中能有效地进行相应酯的热 分解 $\left(100 \sim 120{ }^{\circ} \mathrm{C}\right)$, 通过消除 $\mathrm{CO}_{2}$ 和 $\mathrm{CH}_{3} \mathrm{X}$, 完成相应 的三氟甲基化反应，反应具有条件相对温和、产率高、 试剂便宜易得等优点, 该方法被称为陈试剂(陈方法, Eq. 27), 并广泛被国内外学术界和药业应用于含三氟甲 基的化合物的合成.

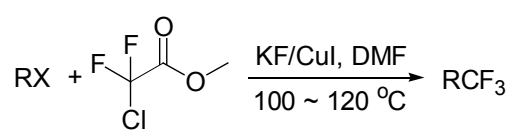

$\mathrm{RX}=\mathrm{Phl}, \mathrm{PhBr}, \mathrm{CH}_{2}=\mathrm{CHCH}_{2} \mathrm{Br}, \mathrm{CH}_{2}=\mathrm{CHBr}$, etc.
2013 年 Senanayake 等 ${ }^{[61]}$ 使用陈试剂, 在研发抗感 染药物中，通过条件优化并使用控制滴加的方法、溶剂 篮选，确定了最佳反应条件,有效控制了放大反应中泡 沫 $\mathrm{CO}_{2}$ 以及三个副产物的形成, 成功制备了关键中间体 (Eq. 28). 值得一提的是，鉴于 1,1-二氟-1-氯代乙酸甲酯 (MCDFA) 在工业生产中大规模生产, 其成本要比使用 Ruppert 试剂降低 $85 \%$.

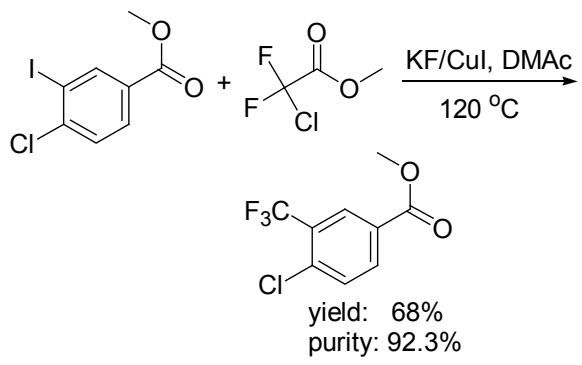

张雯等 ${ }^{[62]}$ 在雅培(Abbott)工作期间，在 COX-2 抑制 剂的新药研发项目中，通过成功抑制卤素交换，制备了 一系列含氟(三氟甲基)的 4,5-二卤代哒嗪酮中间体，对 目标分子进行了系统深入的 SAR 研究, 篎选出高选择 性的临床候选药物, 并有效地大规模制备了 ABT-963 (Scheme 35).

一般而言, 在新药研发中, 为了优化候选药物的物 理化学和药代性质, 多采用后氟化策略. 如 Takeda 制药 的 Zhao 等 ${ }^{[63]}$ 在研发高效 MEK 抑制剂 TAK-733 的工艺 过程中就发现，使用原来的合成方法(Scheme 36), 利用 Selectfluor 进行氟化反应, 产率为 $19 \%$, 而总产率仅为 $3 \%$. 显得不能满足临床研究的需要.

为此他们重新设计了合成路线, 从便宜易得的氟代 丙二酸二甲酯出发，制备了关键含氟中间体，经六步反 应(Scheme 37, 原来是九步反应)有效地制备了候选药 物, 总产率从原来的 3\%提高到 $25 \%$.

在工艺研发 GSK3 3 抑制剂 AZD8027 的过程中, AstraZenca 的 Witt 等 ${ }^{[64]}$ 面临着同样如何有效和方便地引 入氟原子和三氟甲基的问题。很显然，通过用 Selectfluor 引入氟原子后缩合形成氟代嘧啶杂环是极不 可取的(Scheme 38). 一是氟化试剂昂贵, 二是氟化产率 低 $(50 \% \sim 55 \%)$ ，而且有中间体不稳定等缺点.<smiles>CC(C)(O)CCOc1c(-c2ccc(S(C)(=O)=O)cc2)cnn(-c2ccc(F)c(F)c2)c1=O</smiles>

Scheme 35 
<smiles></smiles>

\section{Scheme 36}<smiles>CO[C@H]1COC(C)(C)O1</smiles><smiles>Cn1c(=O)c(F)c(-c2c(F)c(=O)n(C)n(CC3COC(C)(C)O3)c2=O)c2c(=O)n(CC3COC(C)(C)O3)cnc21</smiles>

TAK-733

overall yield: $25 \%$

Scheme 37

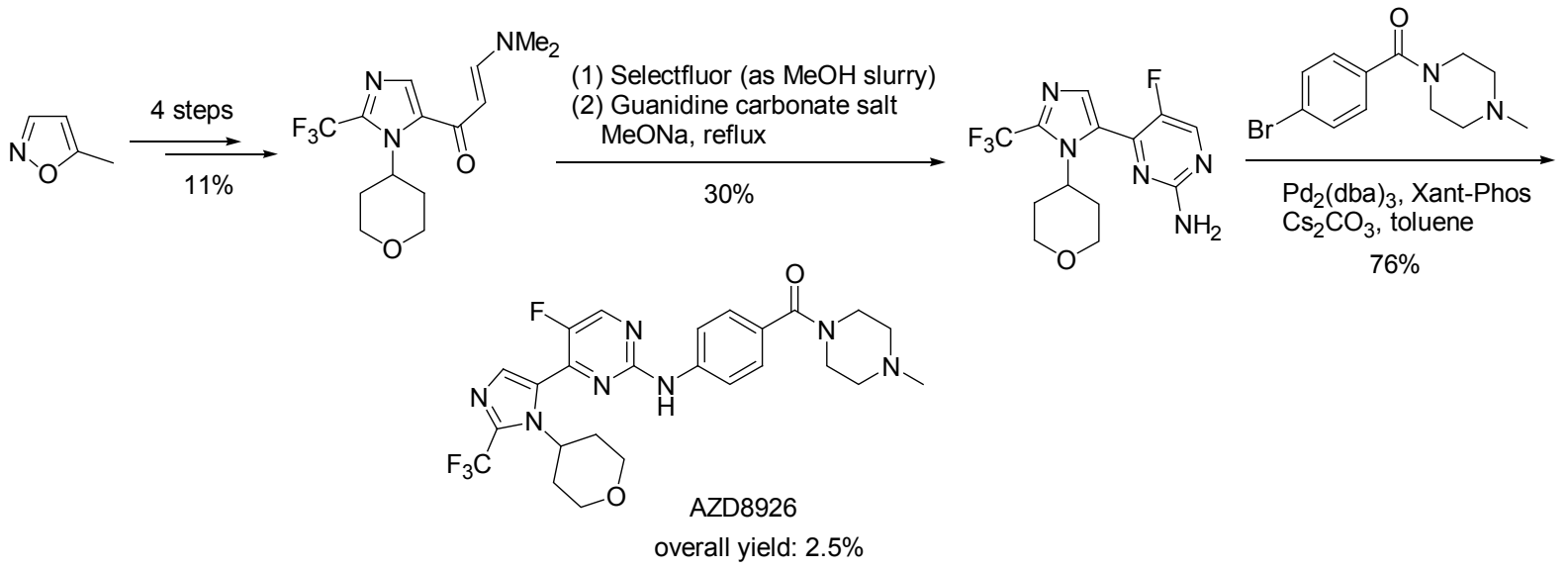

Scheme 38

改进的合成方法首先合成了含氟嘧啶和含三氟甲 基的咪唑片断, 然后通过杂环的 Ziegler 偶联关键反应 等有效制备了 AZD8926, 改进过的合成方法更趋合理 (Scheme 39), 产率更高. 同时把钯催化的胺化反应提前, 避免了消除痕量钯金属所带来的困扰和 API 的损失.

\section{6 总结和展望}

经过这些年的不解努力, 有机氟化学在诸多方面得 到了迅猛的发展, 世界一流的研究小组你追我赶, 创新 立异, 成果频出. 应该承认, 有机金属化学的进展、催化
反应的应用是今天氟化学进展的主推动力. 最近在有机 化学的期刊上很难看到不含有氟化学的论文. 许多有关 氟化学的专著也陆续出版 ${ }^{[65]}$, 综述文章和研究论文也不 断更新 ${ }^{[66]}$.

在这里, 我们将近年来芳香烃氟化反应和芳香烃杂 环化合物 $\mathrm{N}(n=1,2,3)$ 氟甲基化反应的研究进展用图示 的形式进行概括总结 (Schemes 40,41), 并概述了近年来 含氟药物的研究成果. 值得特别强调的是第(III)种反应 类型即新颖的氧化氟化反应(Oxidative fluorination)和氧 化三氟甲基化反应(oxidative trifluoromethylation)是近几 

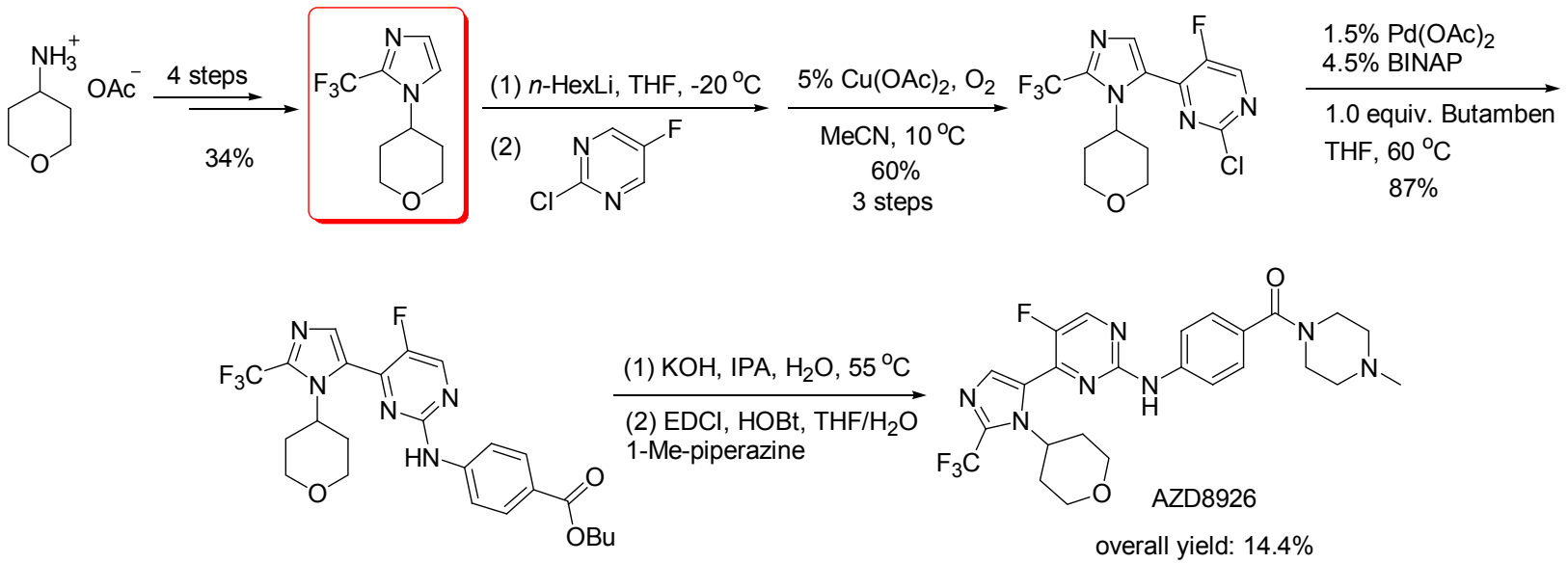

Scheme 39

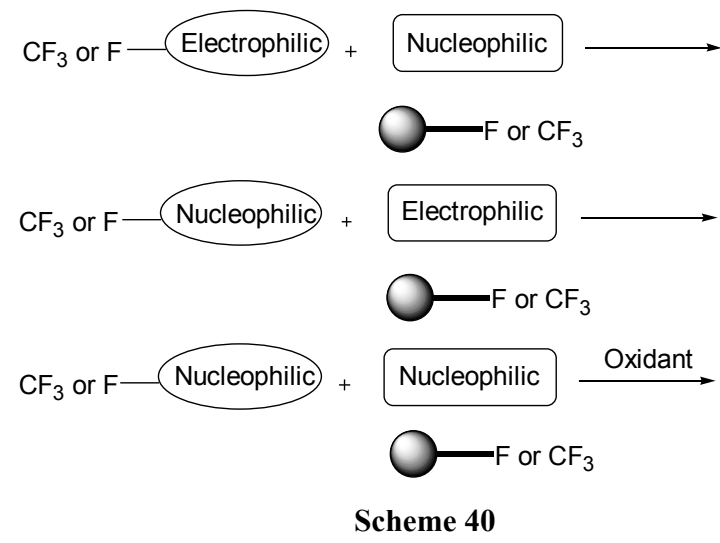

(I)

(II)

(III)

年来的研究热点课题之一. 我们相信最近的氟化学进展 必将为药物研发工作者进行广泛的药物合成研究开辟 了崭新的道路. 毫无疑问, 它必将拓展含氟药物建筑全
新分子的想象空间，提供更多重要的方法和途径.

从发展趋势和方向来看，(1)理想的氟化反应应该是 无金属(Metal-free)参与的或者是高效的过渡金属催化 的过程，应该大力开掘新颖高效的催化系统; (2)从经 济、绿色环保等方面和为工业化放大生产考虑, $\mathrm{Pd}$ 等催 化剂的用量应该尽量控制在 $0.5 \sim 1 \mathrm{~mol} \%$ 之间(目前一般 为 5 10 mol\%左右); (3)氟原子和 $\mathrm{N}$ 氟甲基的引入先后 (现在一般的氟化物原分离纯化较难，区域选择性不好 而附带各种含氟杂质; (4)利用便宜易得的氟试剂，目前 许多含氟试剂较贵；(5)操作简单的工艺，反应应该有不 怕氧不怕水等优点，安全且重复性好; (6)反应应该具有 高产率，尤其是含氟中间体和产物料都是市场上购入含 氟小分子), 理想的状态应该是后加入 $\mathrm{F}$, 目前缺陷是： 收率一般并不太高, 产物纯品纯度不够, 如何进行分离

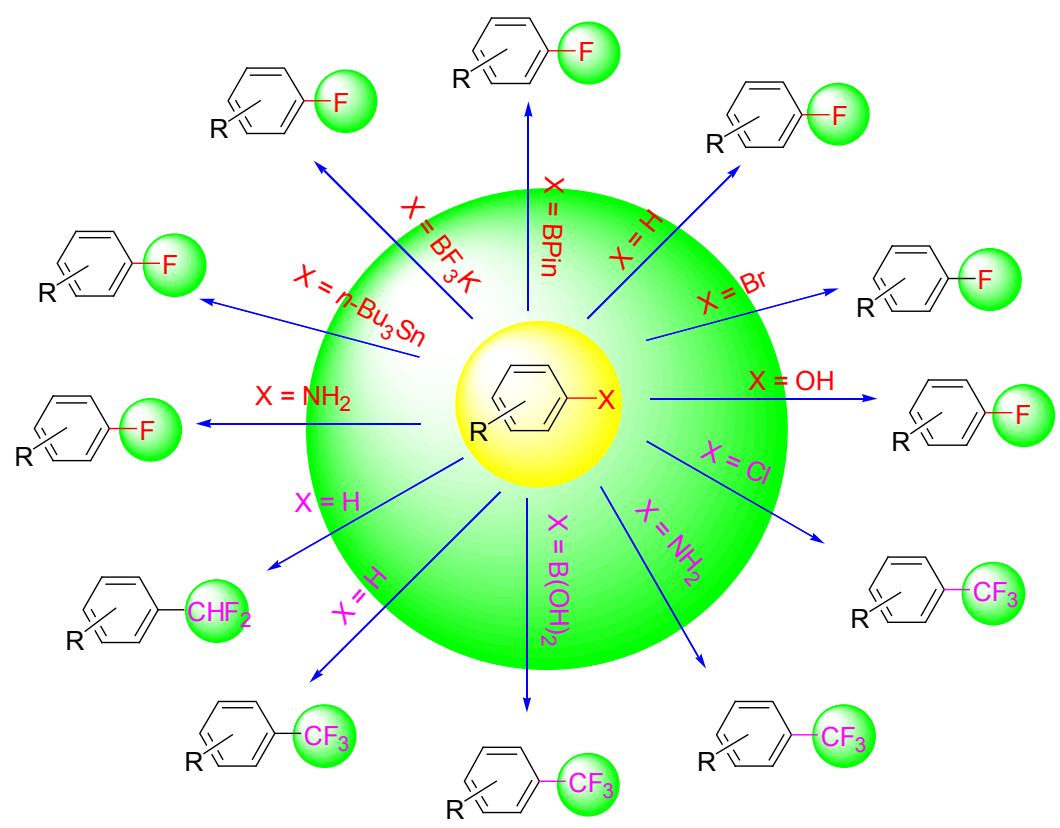

Scheme 41 


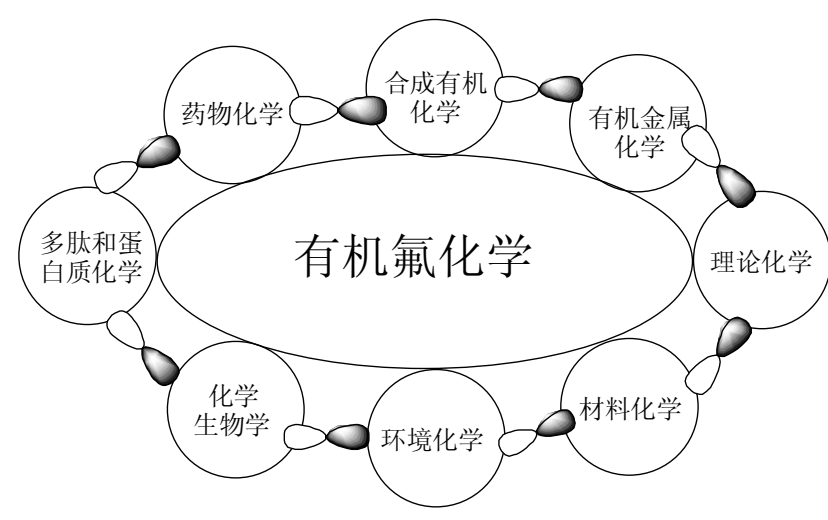

Scheme 42

纯化对工艺开发也是一个很大的挑战, 因为一般而言, 含氟化合物在多数有机溶剂中的溶解度比母体化合物 更佳, 这使得利用重结晶、打浆等手段富积含氟化合物 更趋不可能.

应该指出的是, 目前在候选药物结构单元中什么位 置引入氟原子和三氟甲基团, 仍带有相当的随意性, 仍 然不能利用分子设计和计算化学的方法得到确切的判 断. 也就是说含氟化合物的生理活性与分子结构的构效 关系只能在一定程度上进行预测, 没有形成系统的理论 体系. 显然这也是对药物科研工作者在含氟药物的合成 与创新、开发高效低毒的新型药物提出的挑战.

综上所述, 经过这几年有机氟化学家、有机金属化 学家和药物化学工作者的共同努力, 在许多世界一流实 验室参与和不甘人后的研发热潮中, 有机氟化学的研究 面貌发生了根本性的变化, 极大地推动了含氟药物、多 肽蛋白质化学和化学生物学的发展, 对材料科学和理论 化学的发展也产生了很大的推动(Scheme 42). 毫不夸张 地说，这些年是有机氟化学发展的黄金时代，它所创造 的薮新氟化学反应手段和策略,为药物化学家开拓含氟 药物这座宝藏提供了机遇和创造空间，也激发和丰富了 有机化学家和药物科研人员的创造性和想象力,含氟药 物仍是今后很长一段时间药物研发的一个热点课题, 这 也是摆在中国有机化学家和药物科研人员面前的一大 机遇和挑战.

\section{References}

[1] Wang, J.; Liu, H. Chin. J. Org. Chem. 2011, 31, 1785 (in Chinese). (王江, 柳红, 有机化学, 2011, 31, 1785.)

[2] (a) Wang, J.; Sanchez, M.; Acena, J.; Pozo, C.; Sorochinsky, A.; Fustero, S.; Soloshonok, V.; Liu, H. Chem. Rev. 2014, 114, 2432. (b) Meanwell, N. A. J. Med. Chem. 2011, 54, 2529.

[3] Krik, K. L. Org. Process Res. Dev. 2008, 12, 305.

[4] Ojima, I. J. Org. Chem. 2013, 78, 6358.

[5] (a) Qing, F. L. Chin. J. Org. Chem. 2012, 32, 815 (in Chinese). (卿风翎, 有机化学, 2012, 32, 815.)

(b) Ritter, S. K. Chem. Eng. News 2012, 90, 10. (c) Thayer, A. M. Chem. Eng. News 2006, 84, 15.

[6] (a) Cui, J. J.; Tran, M.; Shen, H.; Nambu, M.; Kung, P. P.; Pairish, M.; Jia, L.; Meng, J.; Funk, L.; Botrous, I.; Mc, M.; Grodsky, N.; Ryan, K.; Padrique, E.; Alton, G.; Timofeevski, S.; Yamazaki, S.; Li, Q.; Zou, H.; Christensen, J.; Mroczkowski, B.; Bender, S.; Kania, R. S.; Edwards, M. P. J. Med. Chem. 2011, 54, 6342.

(b) Li, J.; Cai, Y.; Chen, W.; Liu, X.; Lin, L.; Feng, X. J. Org. Chem. 2012, 77, 9148.

[7] Hansen, K. B.; Hsiao, Y.; Xu, F.; Rivera, N.; Clausen, A.; Kubryk, M.; Krska, S.; Rosnea, T.; Simmons, B.; Balsells, J.; Ikemoto, N.; Sun, Y.; Spindler, F.; Malan, C.; Grabowski, E. J. J.; Armstrong, J. D. J. Am. Chem. Soc. 2009, 131, 8798.

[8] (a) Hagmann, W. K. J. Med. Chem. 2008, 51, 4359.

(b) Ichikawa, M.; Ohtsuka, M.; Ohki, H.; Ota, M.; Haginoya, N.; Itoh, M.; Shibata, Y.; Sugita, K.; Ishigai, Y.; Terayama, K.; Kanda, A.; Usui, H. ACS Med. Chem. Lett. 2013, 4, 932.

[9] Hull, K. L.; Anani, W. Q.; Sanford, M. S. J. Am. Chem. Soc. 2006, $128,7134$.

[10] Wang, X.; Mei, T. S.; Yu, J. Q. J. Am. Chem. Soc. 2009, 131, 7520.

[11] Chan, K. S. L.; Wasa, M.; Wang, X.; Yu, J. Q. Angew. Chem., Int. Ed. 2011, 50, 9081.

[12] Truong, T.; Klimovica, K.; Daugulis, O. J. Am. Chem. Soc. 2013, 135,9342

[13] Ye, Y.; Sanford, M. S. J. Am. Chem. Soc. 2013, 135, 4648.

[14] Fier, P. S.; Luo, J.; Hartwig, J. F. J. Am. Chem. Soc. 2013, 135, 2559.

[15] (a) Furuya, T.; Ritter, T. J. Am. Chem. Soc. 2008, 130, 10060. (b) Mazzotti, A. R.; Campbell, M. G.; Tang, P.; Murphy, J. M.; Ritter, T. J. Am. Chem. Soc. 2013, 135, 14012.

[16] Ye, Y.; Schimler, S. D.; Hanley, P. S.; Sanford, M. S. J. Am. Chem. Soc. 2013, 135, 16292.

[17] Cazorla, C.; Metay, E.; Andrioletti, B.; Lemaire, M. Tetrahedron Lett. 2009, 50, 3936.

[18] Furuya, T.; Ritter, T. Org. Lett. 2009, 11, 2860.

[19] Furuya, T.; Strom, A. E.; Ritter, T. J. Am. Chem. Soc. 2009, 131, 1662.

[20] (a) Tang, P.; Furuya, T.; Ritter, T. J. Am. Chem. Soc. 2010, 132, 12150.

(b) Lee, E.; Hooker, J. M.; Ritter, T. J. Am. Chem. Soc. 2012, 134, 17456.

(c) Gao, Z.; Lim, Y. H.; Tredwell, M.; Li, L.; Verhoog, S.; Hopkinson, M.; Kaluza, W.; Collier, T. L.; Passchier, J.; Huiban, M.; Gouverneur, V. Angew. Chem., Int. Ed. 2012, 51, 6733.

[21] Fier, P. S.; Hartwig, J. F. J. Am. Chem. Soc. 2012, 134, 10795.

[22] Sun, H.; Dimagno, S. G. Angew. Chem., Int. Ed. 2006, 45,2720.

[23] Kumai, S.; Seki, T.; Wada, A. JP 04164068, 1992 [Chem. Abstr. 1992, 117, 233865].

[24] Grushin, V. V.; Marshall, W. J. Organometallics 2008, 27, 4825.

[25] Tang, P.; Wang, W.; Ritter, T. J. Am. Chem. Soc. 2011, 133, 11482.

[26] Wannberg, J.; Wallinder, C.; Unlusoy, M.; Skold, C.; Larhed, M. J. Org. Chem. 2013, 78, 4184.

[27] Fors, B. P.; Watson, D. A.; Biscoe, M. R.; Buchwald, S. L. J. Am. Chem. Soc. 2008, 130, 13552.

[28] Nemoto, H.; Nishiyama, T.; Akai, S. Org. Lett. 2011, 13, 2714.

[29] Adachi, K.; Ohira, Y.; Tomizawa, G.; Ishihara, S.; Oishi, S. J. Fluorine Chem. 2003, 120, 173.

[30] Tian, T.; Zhong, W. H.; Meng, S.; Meng, X. B.; Li, Z. J. J. Org. Chem. 2013, 78, 728 .

[31] Ichiishi, N.; Canty, A. J.; Yates, B. F.; Sanford, M. S. Org. Lett. 2013, 15, 5134.

[32] Olberg, D. E.; Arukwe, J. M.; Grace, D.; Hjelstuen, O. K.; Solbakken, M.; Kindberg, G. M.; Cuthbertson, A. J. Med. Chem. 2010, 53, 
1732.

[33] Hu, J.; Zhang, W.; Wang, F. Chem. Commun. 2009, 45, 7465.

[34] Narjes, F.; Koehler, K. F.; Koch, U.; Gerlach, B.; Colarusso, S.; Steinkuhler, C.; Brunetti, M.; Altamura, S.; De, F. R.; Matassa, V. G. Bioorg. Med. Chem. Lett. 2002, 12, 701.

[35] Chowdhury, M. A.; Abdellatif, K. R. A.; Dong, Y.; Das, D.; Suresh, M. R.; Knaus, E. E. J. Med. Chem. 2009, 52, 1525.

[36] Fujiwara, Y.; Dixon, J. A.; Rodrigue, R. A.; Baxter, R. D.; Dixon, D. D.; Collins, M. R.; Blackmond, D. G.; Baran, P. S. J. Am. Chem. Soc. 2012, 134, 1494.

[37] Fujiwara, Y.; Dixon, J. A.; Hara, F.; Funder, E. D.; Dixon, D. D.; Rodrigue, R. A.; Baxter, R. D.; Herle, B.; Sach, N.; Collins, M. R.; Ishihara, Y.; Baran, P. S. Nature 2012, 492, 95.

[38] Xia, J. B.; Zhu, C.; Chen, C. J. Am. Chem. Soc. 2013, 135, 17494.

[39] Fujikawa, K.; Fujioka, Y.; Kobayashi, A.; Amii, H. Org. Lett. 2011, 13,5560 .

[40] Fier, P. S.; Hartwig, J. F. J. Am. Chem. Soc. 2012, 134, 5524.

[41] Mcmurtrey, K. B.; Racowski, J. M.; Sanford, M. S. Org. Lett. 2012, 14, 4094.

[42] Wang, X.; Zhang, Y.; Wang, J. B. Sci. Sin. Chim. 2012, 42, 1417 (in Chinese).

(王兮，张艳，王剑波，中国科学：化学, 2012, 42, 1417.)

[43] Tomashenko, O. A.; Grushin. V. V. Chem. Rev. 2011, 111, 4475.

[44] (a)Wu, X. F.; Neumann, H.; Beller, M. Chem-Asian J. 2012, 7, 1744.

(b) Furuya, T.; Kamlet, A. S.; Ritter, T. Nature 2011, 473, 470.

(c) Liu, H.; Gu, Z.; Jiang, X. Adv. Synth. Catal. 2013, 355, 617.

[45] Ji, Y.; Brueckl, T.; Baxter, R. D.; Fujiwara, Y.; Seiple, I. B.; Su, S.; Blackmond, D. G.; Baran, P. S.; Baran, P. S. Proceedings of the National Academy of the Sciences of the United States of America, 2011, 108, 14411.

[46] (a) Langlois, B. R.; Laurent, E. R. N. Tetrahedron Lett. 1991, 32, 7525 .

(b) Langlois, B. R.; Laurent, E. R. N. Tetrahedron Lett. 1992, 33, 1291.

[47] (a) Ye, Y.; Kunzi, S. A.; Sanford, M. S. Org. Lett. 2012, 14, 4979. (b) Ye, Y.; Sanford, M. S. J. Am. Chem. Soc. 2012, 134, 9034.

[48] Novak, P.; Lishchynskyi, A.; Grushin, V. V. Angew. Chem., Int. Ed. 2012, 51, 7767.

[49] Dai, J. J.; Fang, C.; Xiao, B.; Yi, J.; Xu, J.; Liu, Z. J.; Lu, X.;Liu, L.; Fu, Y. J. Am. Chem. Soc. 2013, 135, 8436.

[50] Danoun, G.; Bayarmagnai, B.; Grunberg, M. F.; Gooßen, L. J. Angew. Chem., Int. Ed. 2013, 52, 7972.

[51] Wang, X.; Xu, Y.; Mo, F.; Ji, G.; Qiu, D.; Feng, J.; Ye, Y.; Zhang, S.; Zhang, Y.; Wang, J. J. Am. Chem. Soc. 2013, 135, 10330.

[52] Nagib, D. A.; MacMillan, D. W. C. Nature 2011, 480, 224.

[53] Chu, L.; Qing, F. L. J. Am. Chem. Soc. 2012, 134, 1298.

[54] Liu, T.; Shao, X.; Wu, Y.; Shen, Q. Angew. Chem., Int. Ed. 2012, 51,
540.

[55] Zhang, C. P.; Wang, Z. L.; Chen, Q. Y.; Zhang, C. T.; Gu, Y. C.; Xiao, J. C. Angew. Chem., Int. Ed. 2011, 50, 1896.

[56] Mu, X.; Chen, S.; Zhen, X.; Liu, G. Chem. Eur. J. 2011, 17, 6039.

[57] Fu, D.; Zhang, J.; Cao, S. J. Fluorine Chem. 2013, 156, 170.

[58] Zeng, Y.; Zhang, L.; Zhao, Y.; Ni, C.; Zhao, J.; Hu, J. J. Am. Chem. Soc. 2013, 135, 2955.

[59] Wang, X.; Truesdale, L.; Yu, J. J. Am. Chem. Soc. 2010, 132, 3648.

[60] Su, D. B.; Duan, J. X.; Chen, Q. Y. Tetrahedron Lett. 1991, 32, 7689.

[61] Mulder, J. A.; Frutos, R. P.; Patel, N. D.; Qu, B.; Sun, X.; Tampone, T. G.; Gao, J.; Sarvestani, M.; Eriksson, M. C.; Haddad, N.; Shen, S.; Song, J.; Senanayake, C. H. Org. Process Res. Dev. 2013, 17, 940 .

[62] (a) Zhang, J.; Morton, H. E.; Ji, J. Tetrahedron Lett. 2006, 47, 8733. (b) Kerdesky, F. A. J.; Leanna, M. R.; Zhang, J.; Li, W.; Lallaman, J. E.; Ji, J.; Morton, H. E. Org. Process Res. Dev. 2006, 10, 512.

[63] Zhao, Y.; Zhu, L.; Provencal, D. P.; Miller, T. A.; Bryan, C.; Langston, M.; Shen, M.; Bailey, D.; Sha, D.; Palmer, T.; Ho, T.; Li, M. Org. Process Res. Dev. 2012, 16, 1652.

[64] Witt, A.; Teodorovic, P.; Linderberg, M.; Johansson, P.; Minidis, A. Org. Process Res. Dev. 2013, 17, 672.

[65] (a) Ojima, I. Fluorine in Medicinal Chemistry and Chemical Biology, Wiley-Blackwell, 2009.

(b) Petrov, V. A. Fluorinated Heterocyclic Compounds: Synthesis, Chemistry, and Applications, Wiley, 2009.

(c) Roesky, H. W.; Sharpless, K. B. Efficient Preparation of Fluorine Compounds, Wiley, New Jersey, 2012.

(d) Kirsch, P. Modern Fluoroorganic Chemistry: Synthesis, Reactivity, Applications, 2nd, Completely Revised and Enlarged Edition, Wiley-VCH, Weinheim, 2013.

[66] (a) Liang, T.; Neumann, C. N.; Ritter, T. Angew. Chem., Int. Ed. 2013, 52, 8214 .

(b) Studer, A. Angew. Chem., Int. Ed. 2012, 51, 8950.

(c) Grushin, V. V. Acc. Chem. Res. 2010, 43, 160.

(d) Pan, F.; Shi, Z. Acta Chim. Sinica 2012, 70, 1679 (in Chinese).

(潘菲, 施章杰, 化学学报, 2012, 70, 1679.)

(e) He, Z.; Huang, Y.; Verpoort, F. Acta Chim Sinica 2013, 71, 700 (in Chinese).

(何展荣, 黄毅勇, Verpoort Francis, 化学学报, 2013, 71, 700.)

(f) Yang, Y.; You, Z.; Qing, F. Acta Chim. Sinica 2012, 70, 2323

(in Chinese).

(杨义, 游正伟, 卿风悀, 化学学报, 2012, 70, 2323.)

[67] (a) Zhang, J.; Sarma, K. D.; Curran, T. T. Synlett 2013, 0550.

(b) Zhang, J.; Zhang, Y. J. Chin. J. Org. Chem. 2013, 33, 409 (in Chinese).

(张雴, 张英俊, 有机化学, 2013, 33, 409.) 